\title{
SEX-RELATED DIFFERENCES IN VISUOMOTOR SKILL RECOVERY FOLLOWING CONCUSSION IN WORKING-AGED ADULTS
}

\author{
Nicole Smeha
}

\begin{abstract}
A thesis submitted to the Faculty of Graduate Studies in partial fulfillment of the requirements for the degree of

\section{Master Of Science}

Graduate Program in Kinesiology and Health Science York University

Toronto, Ontario

June 2021

(C) Nicole Smeha, 2021 


\begin{abstract}
The performance of complex visuomotor tasks in which the guiding visual information does not align spatially with the required motor output is contingent on the brain's ability to integrate somatosensory information into an appropriate motor response. Performance on such "cognitive-motor integration" tasks is affected in concussion. Here, we investigate the relationship between visuomotor skill performance, concussion history, and sex during the course of a post-concussion management program. Participants with and without a concussion history were tested on their visuomotor skills prior to and following the recovery program. We observed significant visuomotor skill improvement in participants with a concussion history compared to healthy controls. Notably, we observed stronger recovery of skilled performance in females. Our findings indicate that rule-based visuomotor skill is impaired following concussion, though this ability can be improved over the course of a recovery program. Stronger recovery in females suggests sex-related differences in the brain networks controlling skilled performance.
\end{abstract}




\section{Acknowledgements}

I am incredibly thankful to everyone who made this possible:

I would like to thank my wonderful supervisor, Dr. Lauren Sergio, for providing me with the opportunity to engage in research in a meaningful way. Your support, patience, and knowledge were not only encouraging, but made this process both enjoyable and exciting!

I would also like to thank my labmates. A special thank you to Alanna and Alica for always being willing to answer any questions and guiding me through the grad school experience, and to Sijad for your support and going along this journey with me.

Thank you to the Whiplash and Concussion Injury lab for providing me with the ability to participate in research, despite an ongoing pandemic. Thank you to Dr. Loriann Hynes for helping design this study and including a cognitive-motor integration component - this thesis would not have been possible without it. To Leah Henderson, thank you for delivering the manual therapy. Special thank you to Michael Modica and Ravneet Kalkat. To Michael, thank you for handling the data and always being more than happy to help. To Ravneet, thank you for conducting and collecting the assessments that made this thesis possible.

Finally, I would like to thank my family. Without you, I would never have made it this far. 


\section{Table of Contents}

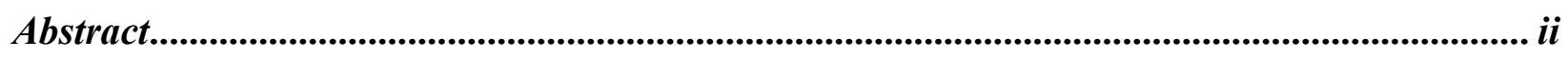

Acknowledgements................................................................................................................

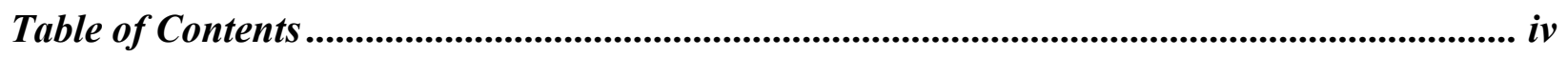

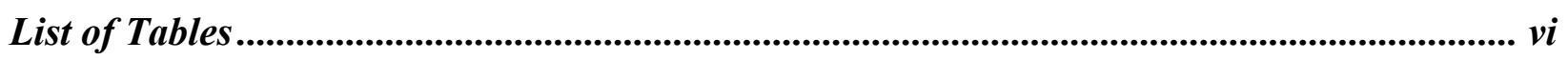

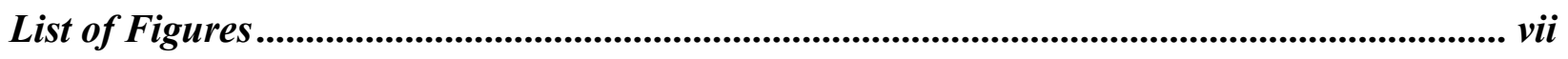

Glossary of Terms ........................................................................................................................ viii

Chapter 1: Introduction and Literature Review ............................................................... 1

Concussion ................................................................................................................................................................. 1

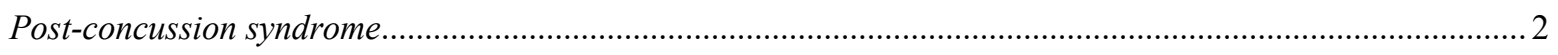

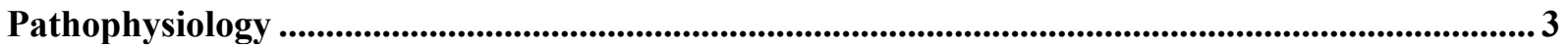

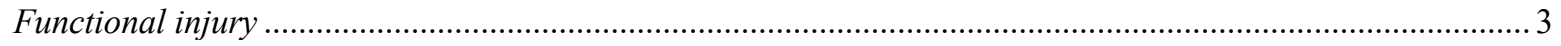

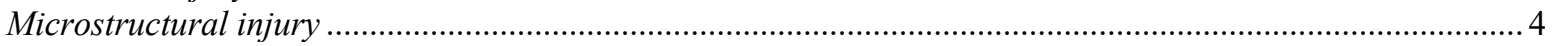

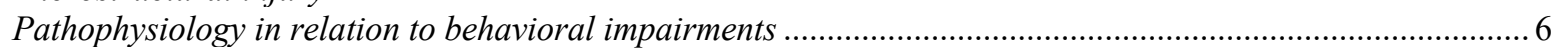

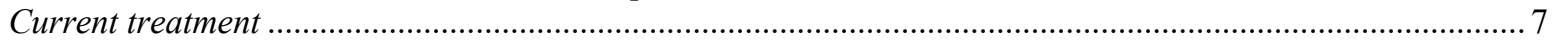

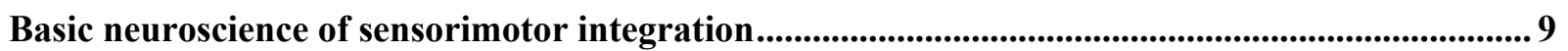

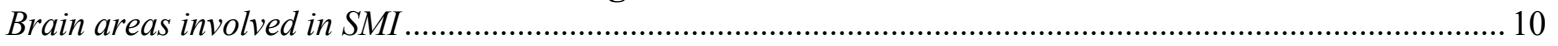

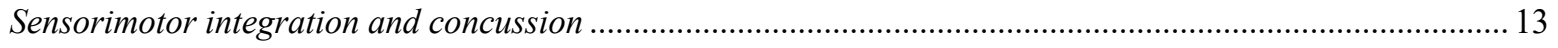

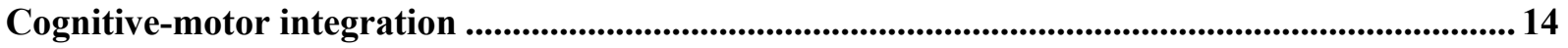

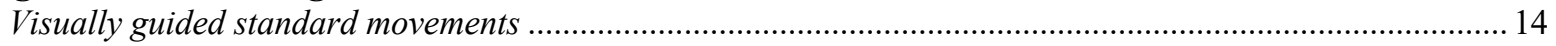

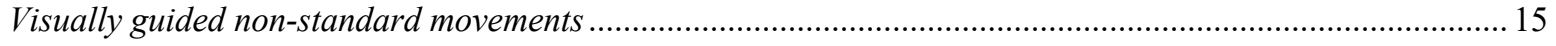

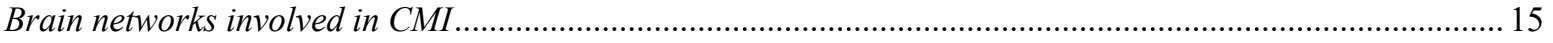

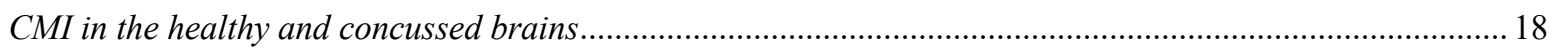

Current study - Purpose and Hypotheses ........................................................................................................ 19

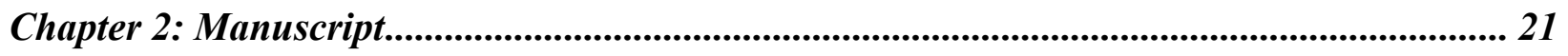

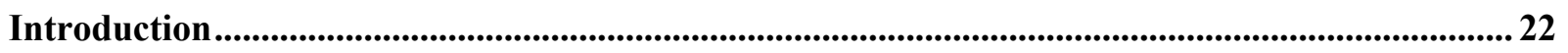

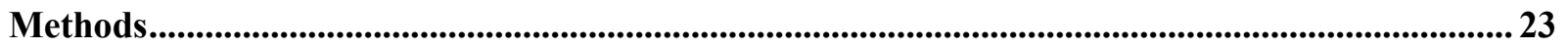

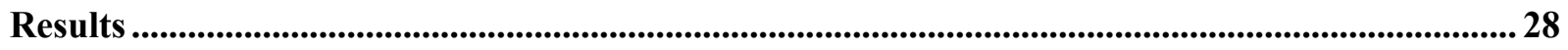

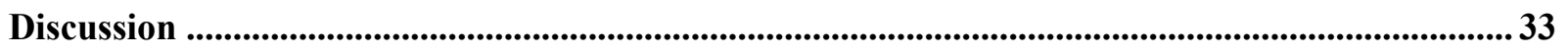

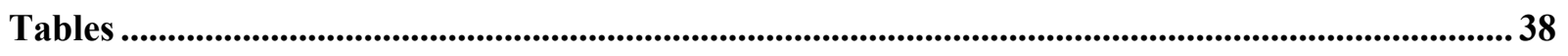

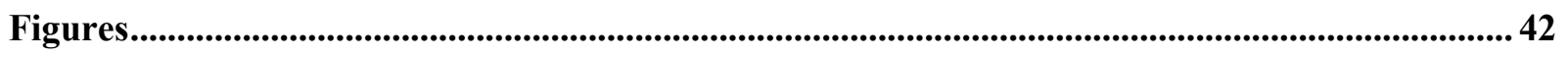

Chapter 3: Discussion \& Conclusions ....................................................................... 52

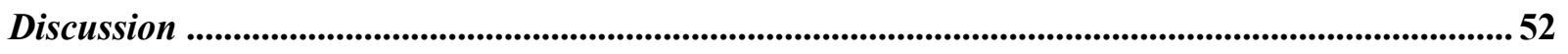

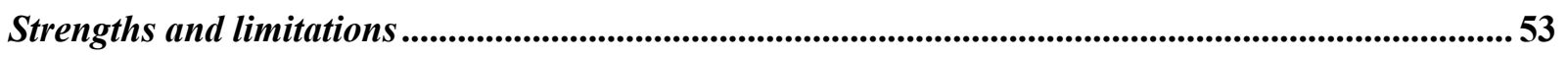

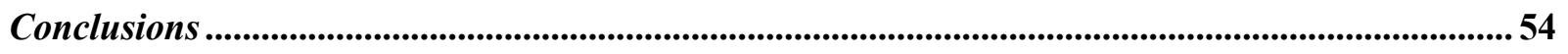

References ........................................................................................................................................ 55

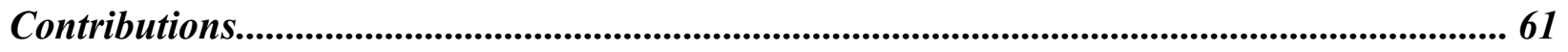

Appendix.......................................................................................................................................62 62 
A : Health Questionnaire................................................................................................................................ 62

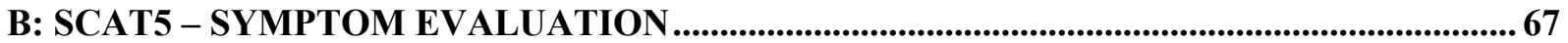




\section{List of Tables}

Table 1: Characteristics for 15 acutely concussed participants, 11 participants with a concussion history, and 17 participants with no history of concussion............................ 38

Table 2: Association between visuomotor performance and history of concussion, sex, and

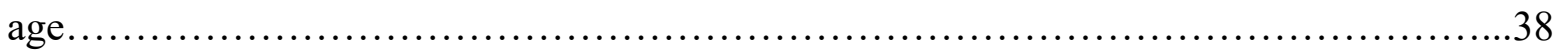




\section{List of Figures}

Figure 1: Research procedure flow diagram and osteopathic techniques provided during this study

Figure 2: Illustration of the tablet-based BrDITM task

Figure 3: Examples of full hand movement trajectories for healthy and acutely concussed participants in the CMI condition.

Figure 4: Percent of trials in the CMI condition resulting in direction reversals, by session and group.

Figure 5: Difference in Movement Time in the CMI and Direct condition, by session and group.

Figure 6: Mean improvement in reaction time over a concussion recovery program in the CMI condition, as a function of number of concussions.

Figure 7: Mean improvements in full and ballistic movement time over a concussion recovery program in the CMI condition, as a function of sex ................................... 48

Figure 8: Mean improvement in peak velocity over a concussion recovery program in the Direct condition, as a function of number of concussions.

Figure 9: Relationship between CMI performance and number of SCAT symptoms

Figure 10: Relationship between CMI performance and SCAT symptom severity score 


\title{
Glossary of Terms
}

\author{
AE - Absolute error \\ AD - Alzheimer's disease \\ BA - Brodmann Area \\ $\mathrm{BG}$ - Basal ganglia \\ BOLD - Blood oxygenation level dependent \\ $\mathrm{BrDI}^{\mathrm{TM}}$ - Brain Dysfunction Indicator \\ CBF - Cerebral blood flow \\ CMI - Cognitive-motor integration \\ CNS - Central nervous system \\ CST - Corticospinal tract \\ CT - Computerized tomography \\ DTI - Diffusion tensor imaging \\ eAD - Early Alzheimer's Disease \\ EEG - Electroencephalography \\ fMRI - Functional magnetic resonance imaging \\ fNIRS - Functional near-infrared spectroscopy \\ LIP - Lateral intraparietal area \\ M1 - Primary motor cortex \\ M1c - Primary motor cortex caudal subregion \\ M1r - Primary motor cortex rostral subregion \\ MCI - Mild cognitive impairment \\ MIP/A5 - Medial intraparietal area/Area 5 \\ MRI - Magnetic resonance imaging \\ MT - Movement time \\ MTf - Total/full movement time \\ $\mathrm{MTb}$ - Ballistic movement time \\ mTBI - Mild traumatic brain injury \\ NSAID - Nonsteroidal anti-inflammatory drug \\ OMT - Osteopathic manual treatment \\ PCS - Post-concussion syndrome \\ PET - Positron emission tomography \\ PL - Path length \\ PLf - Full path length \\ PLb - Ballistic path length \\ PMC - Premotor cortex \\ PMd - Dorsal premotor cortex \\ PPC - Posterior parietal cortex \\ PV - Peak velocity \\ RT - Reaction time \\ S1 - Somatosensory cortex \\ SAI - Short latency afferent inhibition \\ SCAT3/SCAT5 - Sport Concussion Assessment Tool \\ SD - Standard deviation \\ SEF - Supplementary eye-field
}


SLF - Superior longitudinal fasciculus

SMI - Sensorimotor integration

SSRI - Selective serotonin reuptake inhibitor

VE - Variable error

$\% \mathrm{DR}$ - Percentage of direction reversals 


\section{Chapter 1: Introduction and Literature Review Concussion}

Concussion is a form of mild traumatic brain injury (mTBI) induced by biomechanical forces that results in a complex pathological condition affecting the brain (Chancellor et al., 2019; Choe, 2016). An impulsive blow to the head or body triggers this transient neurologic syndrome and produces a constellation of physical and cognitive symptoms, and at times, a loss of consciousness (Chancellor et al., 2019; Sharp \& Jenkins, 2015). Concussion presents a significant health problem and public health concern: 1.6-3.8 million sport-related concussions alone are reported annually in the United States, while it is estimated that 110 per 100, 000 Canadians sustain a concussion annually (Choe, 2016; Gordon et al., 2006; Origenes et al., 2019). However, the prevalence is likely significantly higher: because not all individuals pursue medical care, it is estimated that half of all injuries are unreported (Choe, 2016). Concussion affects all members of society. In younger age groups, sports are the most common cause of concussion. The syndrome accounts for $4-13 \%$ of all injuries ranging from young children to collegiate-level athletes, with an average 2.4 concussions per 10, 000 athletic exposures occurring in this population. In adults, the most common causes of concussion are falls and motor vehicle accidents (Choe, 2016).

Headache is the most commonly reported symptom among a multitude of concussion signs and symptoms. These effects are typically categorized into four domains: somatic (headache, nausea, dizziness), cognitive (difficulty with memory or concentration, mental 'fogginess'), mood (irritability, anxiety, depression), and sleep (too little or too much, difficulty falling asleep) (Choe, 2016; Mullally, 2017). Many patients suffering from concussion experience a gradual resolution of signs and symptoms over three months, although complete recovery can be experienced in the majority of cases by two weeks post-injury (Chancellor et al., 
2019; Mullally, 2017). Despite the optimistic prognosis, several individuals do not recover within this expected timeframe and are described as experiencing persistent symptoms, which may progress into post-concussion syndrome (PCS).

\section{Post-concussion syndrome}

PCS is a perplexing condition: its symptoms are nonspecific, and its cause is unknown. PCS is diagnosed when individuals who have been diagnosed with a concussion persistently suffer from lingering symptoms beyond three months post-injury. The complexities underlying PCS are multifaceted. The syndrome lacks both a diagnostic biomarker and clear clinical guidelines, rendering it vulnerable to misconceptions in the diagnostic procedures (Choe, 2016; Origenes et al., 2019; Sharp \& Jenkins, 2015). Moreover, evidence-based treatment does not exist (Chancellor et al., 2019). Cumulatively, these features render PCS confusing and challenging for both the clinician and the patient.

Several risk factors increase the likelihood of persistent symptoms, including previous history of concussion, more severe initial injury, multiple severe acute symptoms, pre-existing psychological and mood disorders, genetic predisposition, younger age, and the female sex (Fino et al., 2017; Sharp \& Jenkins, 2015). Like concussion, symptoms of PCS are classified within the same four domains, though the condition is typically more debilitating. The duration of symptoms in PCS is indeterminate: symptoms can persist for several years without reliable predictors of outcome or successful resolution (Fino et al., 2017; Sharp \& Jenkins, 2015). The most common persistent symptoms include headache, which has been reported up to 4 years following the initial injury, dizziness, imbalance, and vertigo (Fino et al., 2017; Mullally, 2017). When considering the non-resolving nature of the condition, it becomes particularly evident that these symptoms interfere with one's quality of life and lead to disability. The long-term 
consequences of persistent symptoms and mTBI are an additional concern. Recent studies suggest detrimental long-term effects of concussion and PCS, which may evolve into more drastic neurological conditions with aging (Chancellor et al., 2019; Tremblay et al., 2011).

\section{Pathophysiology}

The disturbances observed following a concussion involve functional and microstructural injuries occurring at the tissue level. Functional injury refers to cellular or physiological dysfunction, including ionic shifts, metabolic changes, and impaired neurotransmission, while microstructural injury refers to physical changes not typically apparent using standard imaging techniques, but may be detectable with more advanced ones (Giza \& Hovda, 2014).

\section{Functional injury}

The acceleration and deceleration forces constituting the concussive event trigger complex neurochemical and neurometabolic cascades (Barkhoudarian et al., 2011). The biomechanical forces disrupt neuronal cell membranes, resulting in extreme alterations in ionic balance. Intracellular potassium exits the cell while an influx of sodium and calcium occurs through voltage-gated channels, culminating in neuronal depolarization (Choe, 2016; Giza \& Hovda, 2014). Depolarization increases the number of open voltage-gated ion channels, creating a feedback loop that ultimately results in the unrestricted flow of ions and further depolarization. This process continues until a widespread relative suppression of neurons is produced, creating a 'depression-like' state - which may be the biological substrate for acute post-concussive impairments (Barkhoudarian et al., 2011; Giza \& Hovda, 2014). The change in membrane potential also results in the widespread and uncontrolled release of several neurotransmitters, particularly excitatory amino acids such as glutamate (Barkhoudarian et al., 2011; Choe, 2016; Giza \& Hovda, 2014). Overall, the combination of these events damages the neurons, further 
increasing the ionic flux and altering astrocytic reuptake mechanisms. As a consequence, proteases are activated, cytokine mediators are released, and reactive oxygen species are produced (Choe, 2016; Giza \& Hovda, 2014).

The alterations in ionic balance caused by the initial injury likewise instigate metabolic deficiencies. In an attempt to reestablish ionic balance, the ATP-dependent ionic pumps begin functioning at maximal capacity. Consequently, the concentration of ADP increases, intracellular energy reserves are exhausted, and neurons begin excessively relying on glycolysis (Giza \& Hovda, 2014). This increased energy demand is ill-timed: following a concussive injury, cerebral blood flow (CBF) is acutely decreased (Choe, 2016). In the acute phase, then, the increased energy demand occurs in an environment with reduced CBF and oxygen-delivery, further uncoupling energy supply and demand (Choe, 2016; Giza \& Hovda, 2014). Eventually, disruption of the Krebs cycle and oxidative phosphorylation results in the production and accumulation of lactate. In more severe cases of concussion, acidosis may occur, leading to the breakdown of the blood-brain barrier (Choe, 2016). In support of the effect of concussion on neuronal metabolism, positron emission tomography scans reveal broad and persistent patterns of hypometabolism following mTBI (Chancellor et al., 2019). These post-traumatic changes in metabolism may be mediated by altered gene expression and transporter regulation following concussion (Giza \& Hovda, 2014).

\section{Microstructural injury}

As previously mentioned, the structural effects of concussion are not generally observable using standard imaging techniques. Rather, structural damage occurs on the cellular level. The biomechanical forces imparted onto neurons and glia can damage axons, dendritic arbors, and astrocytic processes, causing a loss of structural integrity (Giza \& Hovda, 2014). 
Damage to the neuronal cytoskeleton is also involved in the loss of structural integrity: phosphorylation and subsequent collapse of neurofilament side-arms may occur in addition to neurofilament compaction, while axonal stretch disrupts microtubule structure (Barkhoudarian et al., 2011; Giza \& Hovda, 2014). These pathophysiologic processes disrupt axonal transport, reduce neurotransmission, and result in axonal blebbing. In more severe cases, axonal disconnection occurs, causing the accumulation of products in the injured region (Barkhoudarian et al., 2011; Choe, 2016; Giza \& Hovda, 2014). Ultimately, axonal degeneration leads to myelin loss as well as demyelination of intact axons, further impairing signal conduction and leaving axons more vulnerable to injury (Choe, 2016; Giza \& Hovda, 2014).

The combination of these factors triggers a local inflammatory response mediated by microglial infiltration to the injured area. Inflammatory cells scavenge cellular debris and create a barrier between damaged and intact tissues by extending processes that form direct contact with the damaged axons (Choe, 2016). The aforementioned release of cytokine mediators, proteases, and reactive free radical species also contributes to the inflammatory process. In addition to this central nervous system (CNS) immune response, peripheral immune cells accumulate in the injured area as a consequence of increased permeability through the blood brain barrier and vascular changes (Choe, 2016). Notably, it is currently unclear whether the inflammatory response is protective, with the aim of repairing and restoring damaged structures, or if it is the reduction of the inflammatory response that allows for restitution (Choe, 2016).

Structural damage is generally imperceptible when standard imaging techniques, including computerized tomography (CT) and structural magnetic resonance imaging (MRI) scanners, are utilized. However, many studies have documented post-traumatic structural abnormalities that are detectable using functional techniques, such as functional MRI (fMRI), 
positron emission tomography (PET), and diffusion tensor imaging (DTI) (Chancellor et al., 2019). The use of fMRI post-concussion reveals a general trend of reduced blood oxygenation level dependent (BOLD) signaling in the frontal regions of the brain, in addition to white matter alterations detected by DTI (Chancellor et al., 2019; Tremblay et al., 2011). However, these imaging modalities cannot yet be used for diagnostic purposes; the predictive value of these markers is not evident with regard to the evaluation and diagnosis of heterogeneous individual injuries (Chancellor et al., 2019).

The etiologies of prolonged concussion symptoms and PCS are much less understood, though it is suggested that persistent symptoms may be due to the aforementioned alterations in neuronal circuitry and neurotransmission (Barlow et al., 2014).

\section{Pathophysiology in relation to behavioral impairments}

The metabolic changes, molecular cascades, and microstructural injuries engender observable cognitive and behavioural repercussions. Alterations in glucose metabolism are associated with spatial learning impairments, which may further deteriorate with aging and result in insufficient cerebral function (Barkhoudarian et al., 2011; Chancellor et al., 2019). Similarly, axonal injury and white matter tract changes are associated with several cognitive deficits, including impairments in awareness, processing speed, memory, attention, and executive function (Barkhoudarian et al., 2011; Białuńska \& Salvatore, 2017; Giza \& Hovda, 2014; Sharp \& Jenkins, 2015). In fact, changes in the corpus collosum observed using DTI have been correlated with impaired performance during cognitive testing (Barkhoudarian et al., 2011; Giza \& Hovda, 2014). Moreover, abnormal increases in BOLD activity have been reported several days to weeks following concussion in adults and older adolescents, and these changes have been correlated with impaired performance on cognitive tests. In contrast, younger adolescents 
demonstrate worse working memory performance associated with significantly reduced taskrelated BOLD activity (Giza \& Hovda, 2014).

Behavioral impairments, such as performance decrements in reaction time and motor speed, have likewise been associated with changes in DTI in subcortical areas, such as the inferior frontal, superior frontal, and supracallosal areas (Barkhoudarian et al., 2011; Giza \& Hovda, 2014; Sharp \& Jenkins, 2015).

\section{Current treatment}

Concussion and PCS affect both the individual who suffers an injury and society as a whole. The broad range of symptoms, in combination with non-resolving symptoms, can quickly lead to both conditions becoming debilitating. Moreover, the high incidence and cost have repercussions on a societal level. Yet, no treatment exists for this injury. Equally concerning, studies investigating the treatment of chronic mTBI symptoms are scarce (Choe, 2016; Fino et al., 2017).

Current recommendations prescribe patients complete physical and cognitive rest for an undefined period of time until symptoms subside, and they can return to their daily activities (Choe, 2016). However, more recent studies suggest that while cognitive and physical rest are advisable for the first 1-5 days post-injury, early physical activity and moderate cognitive activity engaged in as tolerated are associated with lower rates of persistent post-concussive symptoms (Mullally, 2017). Following rest, individuals undergo a sequence of physical and cognitive tests, and if cleared by a physician, are told to return to their work, school, or sport. It is important to note that the current tools used by clinicians to determine recovery are inadequate: they test one domain at a time or rely on self-report, leading many individuals to return to work or sport with impairments that may leave them vulnerable to further injury 
(Hurtubise et al., 2016; Sergio et al., 2020). In fact, recent studies have shown that the commonly used assessment tools, such as neurocognitive testing and the Sport Concussion Assessment Tool (SCAT3), may not be sensitive enough to determine if athletes are safe to return to sport (Hurtubise et al., 2016). Testing one domain at a time is of particular concern: commonly used tests evaluate physical and cognitive abilities sequentially, despite the fact that the integration of cognition and motor action, known as cognitive-motor integration (CMI), is essential to successful interactions with our environments.

Furthermore, cognitive and physical rest may not be feasible for those experiencing persistent symptoms in the long-term, and their ability to successfully pass the assessments that permit them to return to normal life is uncertain. The lack of evidence from randomized-control trials to assist clinicians in treating lingering post-concussion symptoms limits those suffering from physical pain, neurocognitive deficits, and mood disturbances to symptomatic treatment (Choe, 2016; Mullally, 2017).

In general, non-pharmacologic measures are preferred, particularly in the acute phase. If the patient struggles with mood disturbances, such as anxiety or depression, behavioral therapies are prioritized, while compensatory measures such as memory logs are utilized for challenges with memory and related symptoms (Origenes et al., 2019). Patients experiencing sleep dysfunction are advised to practice good sleep hygiene. Even in the subacute and chronic stages, pharmacologic options are only considered once conservative approaches fail. Simple analgesics, including nonsteroidal anti-inflammatory drugs (NSAIDs), are the primary treatment for posttraumatic headache (Mullally, 2017). If they are not effective, triptans can be prescribed, particularly for headaches with migranous features. Antiemetics can be prescribed for nausea (Mullally, 2017). If practicing good sleep hygiene is ineffective, melatonin is frequently 
recommended, though stronger sleep aids can be prescribed if necessary (Barlow et al., 2014).

Similarly, if behavioral therapies are ineffective, selective serotonin reuptake inhibitors (SSRIs) are the preferred medications for anxiety and depression, while neuro-stimulants, dopaminergic agonists, and attention deficit medications are the most common pharmacologic measures for deficits in attention (Barlow et al., 2014). It is important to note that these measures are used without conclusive evidence supporting their efficacy as treatments for concussion either acutely or chronically; rather, they are used on a trial-and-error basis. In addition, clinicians are provided with little guidance for the management of significantly debilitated patients and patients with persistent symptoms.

\section{Basic neuroscience of sensorimotor integration}

Our ability to interact with our environment is made possible through a process known as sensorimotor integration (SMI). SMI refers to the process through which we incorporate sensory inputs with information about our bodies and the external environment in order to inform coordinated motor outputs (Edwards et al., 2019). In the case of goal-directed hand movements, sensory inputs provide information in an egocentric reference frame. Information about the location, weight, size, and shape of an object is incorporated into this reference frame. To accurately incorporate somatosensory feedback from the periphery into the motor cortex and successfully compute the correct motor output, kinetic and trajectory information about the hand and upper extremities is also needed (Brown et al., 2018; Edwards et al., 2019). Goal-directed movements of the hand are of primary interest because these kinds of movements are the ones required to perform most tasks of daily living - for instance, tying one's shoes or typing (Edwards et al., 2019). Evidently, these highly coordinated voluntary movements involve interacting with and manipulating objects in the environment, and are thus are contingent on 
SMI. Integrating sensory information into the motor cortex allows us to generate the most efficient motor plan and optimize current and future performance (Edwards et al., 2019).

\section{Brain areas involved in SMI}

The learning of a motor skill and its effective performance requires the coordination of multiple brain areas. In the human brain, these areas include the primary motor cortex (M1), the somatosensory cortex (S1), and the posterior parietal cortex (PPC). Non-cortical structures involved include the basal ganglia (BG), the cerebellum, the brainstem, and the spinal cord (Edwards et al., 2019; Matur \& Öge, 2017). Sensory signals from the PPC and S1 convey relevant information about somatosensation, proprioception, and visuomotor transformations to the M1 (Edwards et al., 2019). The PPC and S1 are indispensable: these areas integrate sensory with motor information at the level of the cortex and contribute to the corticospinal tract, thus permitting the execution and control of skilled hand movements used routinely in daily life (Edwards et al., 2019). The M1 uses the transformed sensory information about the location and space of the internal and external world to inform motor commands (Edwards et al., 2019). The roles of the M1, S1, and PPC in SMI will now be examined in depth.

The $S 1$ is fundamental to our ability to integrate sensory information with motor commands; it is involved in the processing of proprioceptive, tactile, and nociceptive information (Edwards et al., 2019). S1 is somatotopically organized: representations of body regions are localized to specific cortical areas. It is composed of Brodmann areas (BA) 3a, 3b, 1, and 2, and receives somatosensory input from the thalamus. Somatosensory information is relayed from the periphery to the thalamus from the medial lemniscus through the spinothalamic tract. If the peripheral sensory information is task-irrelevant, it can be filtered out through the inhibition of afferent pathways in a process termed sensory gating. The thalamic nuclei are essential for 
sensory gating, with the posterior medial nucleus of the thalamus in particular connecting to inhibitory neurons from other cortical layers (Edwards et al., 2019). In addition to connections from the thalamus, $\mathrm{S} 1$ projects directly to the M1, relaying somatosensory information to this area and allowing ongoing sensory input to be used to refine and update descending motor commands (Edwards et al., 2019).

M1. The execution of voluntary movements would not be possible without the M1. The most abundant projections from M1 are to motor neurons that innervate hand muscles, accordingly enabling the direct and individualized control of fingers that is required for skilled hand movements (Edwards et al., 2019). These projections are primarily comprised of pyramidal neurons in layer 5; the axons of these neurons are bundled together and form a significant portion of the corticospinal tract (CST). Of these neurons, $85-90 \%$ of the fibers in each respective hemisphere decussate to provide control of the contralateral hand, while the remaining fibers maintain ipsilateral projections and have a minor role in distal extremity motor control (Edwards et al., 2019). Although there are other pathways involved in hand function, the CST is the largest contributor to skilled hand movement. Motor commands can be delivered to alpha-motor neurons directly from corticomotoneuronal cells. Indirect control of movement is possible through neurons that synapse onto interneurons in intermediate zones (Edwards et al., 2019; Matur \& Öge, 2017). The importance of the CST's role in connecting M1 to the alpha-motor neurons innervating the muscles of the hand is exhibited by the fact that lesions to these axonal fibers specifically causes a loss of individualized finger functions.

Like S1, M1 is also topographically organized. Notably, there is a larger spatial representation for the hands compared to other areas, reflecting the relative importance of the output from corticomotoneuronal cells to the hands. The M1 hand area is separated into caudal 
(M1c) and rostral (M1r) subregions: corticomotoneuronal cells primarily arise from M1c and provide direct control of hand and distal forearm movements, while neurons in M1r influence motor control indirectly using interneurons in the spinal cord (Edwards et al., 2019). S1 has strong reciprocal connections with M1c, while the PPC has weaker connections to M1r.

Cells in M1 fire differentially to allow for a variety of hand functions: individual neurons can preferentially code for single or multiple fingers or more proximal joints, and the kinematics and kinetics of a movement, including direction, speed, and force, are also encoded (Edwards et al., 2019).

Once representations of the external environment have been generated from visual, proprioceptive, and tactile input, these representations are combined with internal representations of the motor system, such as hand position, to create an internal model. The individual's internal model will also be influenced by prior motor executions, which contributes to the development of an efference copy of the motor output. Using relevant sensory information, the M1 then generates a motor command. Based on the sensory information and the efference copy, predictions about the expected sensory feedback that will result from the motor command are generated. If the sensory reafferent information is inconsistent with the predicted feedback, the model will be adapted and the error will be corrected by adjusting the motor command. Evidently, though it is critical to movement, the M1 cannot function independently.

The $P P C$ is central to SMI. It is composed of BA 5, 7, 39, and 40, and has connections to M1 and the premotor cortex (PMC) via the superior longitudinal fasciculus (SLF) (Edwards et al., 2019). The PPC is a multisensory association area responsible for the integration of multiple sensory modalities. This area is functionally separated; the rostral portion of the PPC is 
connected to somatosensory and motor regions, while the caudal portion has connections with sensory areas (Edwards et al., 2019).

The PPC receives direct reciprocal inputs from the dorsomedial visual area, providing continuous visual motion analysis. Moreover, sensory information from proprioceptive and tactile activity of hand movements is provided to PPC from somatosensory area S2 and the parietal ventral area, in addition to weaker inputs from S1 (Edwards et al., 2019). Altogether, these inputs contribute to the sensorimotor processing that is required for skilled hand movements. The visual information received by BA 5 is critical because this area is responsible for visuomotor transformations, while the proprioceptive and tactile information guides hand exploration and object discrimination (Edwards et al., 2019). These sensory signals are integrated into reference frames and complex sensorimotor representations by the PPC, to be relayed to the M1. Based on this information, the M1 can optimize motor commands, including visuomotor control (Edwards et al., 2019). PPC neurons are therefore involved in the control and error correction of a movement once it is initiated, and are important for movement planning. The PPC is indisputably important during the SMI required for successfully performing goaldirected hand movements (Edwards et al., 2019).

\section{Sensorimotor integration and concussion}

SMI ability is altered by brain injury. Studies have demonstrated reductions in short latency afferent inhibition (SAI), a marker of cholinergic activity in SMI, following severe traumatic brain injury (Tremblay et al., 2011). SAI is a marker of cholinergic activity that is found in diverse pathologies that share motor and memory dysfunctions similar to those found in PCS, such as Parkinson's disease (Tremblay et al., 2011). Likewise, SAI interacts with concussion-vulnerable inhibitory circuits of M1 for SMI. Moreover, visuomotor assessments 
following concussion reveal poorer visuomotor integration at 10 days, 30 days, and greater than 30 days post-injury, suggesting that visuomotor deficits persist for much longer than physical symptoms (Baker \& Cinelli, 2014; Slobounov et al., 2006). It is thus of interest to assess SMI post-concussion and attempt to rehabilitate it. Visuomotor integration is the form of SMI that will be investigated in the current study.

\section{Cognitive-motor integration}

\section{Visually guided standard movements}

As mentioned above, our daily interaction with objects requires the simultaneous use of the eyes and hands. Typical movements involve direct interaction with an object of interest: the visual stimulus is also the target of the action (Hwang et al., 2014). In these standard visuallyguided reaching movements, a saccade towards a target object occurs before reaching is initiated. Foveation of the object provides visual information about its position and shape, thus improving accuracy of the reach (Granek et al., 2012; Hwang et al., 2014). Foveation of the object allows for improved accuracy of arm reaching because control between the eyes and the arms is linked in the brain: the neural pathways underlying the control of the two effectors share information (Gorbet \& Sergio, 2018; Hwang et al., 2014). In fact, eye-hand coordination relies on the interaction between the saccade and reach control pathways (Hwang et al., 2014). The exchange of spatial and temporal information between these two pathways allows for the visual information about the spatial location of the target to be translated into an appropriate set of muscle activation patterns (Crutcher et al., 2004; Hwang et al., 2014). Thus, an accurate reach is produced. This interpathway communication has been suggested to rely on the PPC (Crutcher et al., 2004). Moreover, neural representations of target location, hand trajectory, joint kinematics, 
and joint dynamics are suggested to reside in the M1, PMC, putamen, and supplementary motor area (Crutcher et al., 2004).

\section{Visually guided non-standard movements}

Just as visual to motor transformations are required in standard reaching movements, nonstandard visuomotor tasks also consist of visual to motor transformations. However, in nonstandard reaching movements, the relationship between the eyes and the arm is indirect: rather than the visual information from the object guiding the movement of the arm towards the same spatial location, the guiding visual information is spatially decoupled from the arm movement (Hurtubise et al., 2016; Hwang et al., 2014). Non-standard tasks decoupling vision and action often involve two components: explicit task rules and sensorimotor recalibration. Because the eyes move in a different direction or in a different plane than the movement of the arm, the integration of context specific rules into the planning of reaching movements is necessary (Gorbet \& Sergio, 2019). In addition to this requirement for cognitive-motor integration, sensorimotor recalibration is also required. When the eyes and arm are decoupled, the proprioceptive and motor components of a reaching task are no longer consistent with the guiding visual information. The relationship between the gain of visual feedback relative to the distance moved by the arm can change as well (Dalecki, Gorbet, \& Sergio, 2019; Gorbet \& Sergio, 2018). Thus, in non-standard tasks, visuomotor mappings between the stimulus and the response must be learned and adjusted (Hurtubise et al., 2016; Hwang et al., 2014; Sergio et al., 2009).

\section{Brain networks involved in CMI}

Though non-standard reaching tasks require learning, standard tasks tend to be more innate, learned in infancy (Piaget, 1965). As previously mentioned, the PPC is a critical component of the pathways that link the eyes and the arm and result in the production of standard reaching 
movements. The shared circuitry between the two effectors prevents the need to independently plan the movement of the eyes and the arm, rendering these movements easier to perform and process than non-standard ones (Gorbet \& Sergio, 2016). More specifically, one hypothesis suggests that the PPC's role in the default coupling of the eyes and the arm relies on two areas within it: the medial intraparietal area/Area 5 (MIP/A5) and the lateral intraparietal area (LIP) (Hwang et al., 2014). The hypothesis posits that the LIP transmits saccade onset time information to the reach control pathway in the MIP/A5 (Hwang et al., 2014). The communication between these two areas may occur within the PPC or through downstream cortical areas (Hwang et al., 2014). Ultimately, communication between the LIP and MIP/A5 generates coherent activity in both areas, allowing for the coordinated temporal coupling which facilitates looking and reaching (Hwang et al., 2014). However, the default coupling of the eyes and the arm does not rely solely on the PPC. The supplementary eye-field (SEF) and parietofrontal networks likewise aid in linking the control of reaches and saccades (Gorbet \& Sergio, 2019; Hawkins et al., 2013).

In contrast, as tasks become increasingly dissociated, reduced activity is observed in these areas. Non-standard reaching would presumably require inhibition of the networks automatically coupling the eyes and the arm (Sergio et al., 2009); this may account for the reduced activity that is observed. The inhibition of default eye-hand coordination networks in non-standard tasks is accompanied by a simultaneous increase in attention and tool-related areas, such as in the prefrontal cortex, the dorsal premotor cortex (PMd), and nuclei of the basal ganglia (BG) (Sergio et al., 2009; Wise et al., 1996). Sensory information from various modalities, including vision and proprioception, is integrated and sent forward from the parietal cortex to the PMd (Sergio et al., 2009). This information is used by the PMd to select an appropriate motor response within the context of the non-standard visuomotor task and plan reaching (Sergio et al., 2009). The PMd's 
role in the production of non-standard movement control could be temporal: in tasks spatially dissociating the location of visual cues and reaching responses, the activity of individual PMd cells initially coincides with the direction of the visual cue, but later coincides with the direction of the movement target prior to arm movement initiation (Gorbet \& Sergio, 2009). This suggests the PMd may be taking contextual information provided by a visual cue and transforming it into signals for the patterns of muscle activity required to achieve a correct motor response (Sayegh et al., 2017; Sergio et al., 2009). The BG play a critical role in this process: it may provide information to the PMd about the appropriateness of a particular cue-response pairing (Sergio et al., 2009). The ventral and orbital components of the prefrontal cortex are also crucial for the performance of nonstandard mapping tasks (Murray et al., 2000).

Altogether, the performance of non-standard reaching tasks depends on intact connections between the frontal, parietal, and subcortical brain regions (Hurtubise et al., 2016; Sayegh et al., 2017). The activity in these areas comprises two pathways. In the first pathway, which involves the dorsal premotor and parietal cortices, sensory information is integrated with spatial awareness, and the necessary transformations for sensorimotor recalibration are performed (Sergio et al., 2009). In the second pathway, which involves the parietal and prefrontal areas, sensory information is integrated with explicit and cognitive information to guide action (Granek \& Sergio, 2015). Ultimately, both pathways are joined and united in the M1 and spinal cord, where the dynamic factors and biomechanical details needed for final movement production are incorporated. The initiation and production of the final dissociated reaching movements rely heavily on cerebellarcortical and BG-cortical loops (Sergio et al., 2009).

Evidently, tasks dissociating the movement of the eyes and arm are complex. Parietofrontal networks are essential to cognitive-motor associations, transformational movements, and 
understanding the spatial relationships between the two effectors. There is, however, another aspect to non-standard visuomotor mapping. Non-standard situations can include reaching along a different spatial plane. Reaching on a horizontal plane while viewing guiding visual information on a frontal plane activates regions in the dorsal premotor cortex and the superior parietal cortex (Gorbet \& Sergio, 2019). Plane dissociation requires the transposition of a motor plan onto another location, and is commonly experienced in daily tasks (Hurtubise et al., 2016). The use of a computer mouse, for instance, involves hand movement in the horizontal plane while the visual target moves in the frontal plane. In addition to the transposition of a motor plan onto another location, tasks performed on a different plane also require CMI - individuals must learn explicit rules (Gorbet \& Sergio, 2018). For instance, they must learn the rule that moving the mouse forward horizontally moves the pointer upward on the screen. When visuomotor rotations are combined with a plane-change, two levels of decoupling of vision and motor action occur. While simultaneously transposing a motor plan onto another location, individuals must successfully adapt to a situation which requires a mental rotation to realign the hand movement relative to the visual target (Granek et al., 2012; Hurtubise et al., 2016). The combination of these two non-standard aspects of a task renders the performance of reaching in these situations challenging.

\section{CMI in the healthy and concussed brains}

Previous research demonstrates declines in performance when any level of non-standard mapping is introduced, whether it be visual feedback rotation, spatial plane change, or both (Sergio et al., 2009). These deficits are found in healthy and patient populations. Previous research in our lab examining healthy, young individuals without any history of neurological problems has demonstrated that dissociating gaze and reach leads to changes in kinematic properties such as hand-path curvature, eye reaction time, peak eye velocity, and arm movement time when compared 
to standard tasks (Gorbet \& Sergio, 2009). However, adults with early Alzheimer's disease (eAD) and mild cognitive impairments (MCI) experience more drastic performance declines in nonstandard tasks compared to healthy adults. Similarly, when dissociation is introduced between the guiding visual information and the required motor action, our lab has demonstrated performance differences in varsity-level athletes, adolescents, and children with a history of concussion compared to healthy age-matched controls (Brown et al., 2015; Dalecki et al., 2016; Dalecki, Gorbet, \& Sergio, 2019; Hurtubise et al., 2016). These differences include impaired movement planning and execution, as demonstrated by significant group differences found in reaction time, movement time, and precision (Brown et al., 2015; Hurtubise et al., 2016). It is important to note that in individuals with a history of concussion, these deficits in both motor planning and execution also existed in the performance of standard tasks, albeit to a lesser extent than in the non-standard tasks decoupling vision and action (Hurtubise et al., 2016).

Thus, there appears to be an impaired ability to integrate rules into coordinated motor tasks with the presence of clinically altered brain function. Non-standard tasks are an important aspect of our daily lives: these tasks are critical for the successful use of a computer, playing a video game, operating machinery remotely, or driving a car. It is therefore important to understand how the timing and kinematics of non-standard tasks compare to those in standard ones following concussion, the possible neural mechanisms underlying the differences, and whether CMI performance can be improved when a therapeutic program is introduced.

\section{Current study - Purpose and Hypotheses}

Ensuring that skilled performance has recovered following a concussive injury is important in that it will decrease vulnerability to another injury, and return an individual to their previous quality of daily life. The main aim of the present study was, therefore, to examine the 
recovery of CMI performance in concussed working-aged adults and adults with a history of concussion compared with healthy no-history controls during the course of a post-concussion management program. A secondary aim of this study was to examine whether sex- and agerelated differences were related to CMI performance over the duration of the program.

In accordance with previous findings, we hypothesize that using a CMI task will expose performance deficits in concussed individuals compared to healthy controls. Following a 6-week concussion management program, we predict that CMI performance in individuals affected by concussion will be improved. As an exploratory aspect to this study, we will examine the relationship between visuomotor skill performance, concussion history, and sex during the course of a post-concussion management program, in addition to the relationship between CMI performance and symptom recovery. 
Chapter 2: Manuscript

SEX-RELATED DIFFERENCES IN VISUOMOTOR SKILL RECOVERY FOLLOWING CONCUSSION IN WORKING AGED ADULTS 


\section{Chapter 2: Manuscript}

\section{Introduction}

Concussion is a form of mild traumatic brain injury (mTBI) induced by biomechanical forces that results in a complex pathophysiological condition affecting the brain (Chancellor et al., 2019; Choe, 2016). An impulsive blow to the head or body triggers this transient neurologic syndrome and produces a constellation of physical and cognitive symptoms, and at times, a loss of consciousness (Chancellor et al., 2019; Sharp \& Jenkins, 2015). Concussion presents a significant health problem and public health concern: 1.6-3.8 million sport-related concussions alone are reported annually in the United States, while it is estimated that 110 per 100, 000 Canadians sustain a concussion annually (Choe, 2016; Gordon et al., 2006; Origenes et al., 2019). Many patients suffering from concussion experience a gradual resolution of signs and symptoms over three months, although complete recovery can be experienced in the majority of cases by two weeks post-injury (Chancellor et al., 2019; Mullally, 2017). Despite the optimistic prognosis, several individuals do not recover within this expected timeframe and are described as experiencing persistent symptoms, which may progress into post-concussion syndrome (PCS); these symptoms may interfere with one's quality of life and lead to disability.

Current recommendations prescribe patients complete physical and cognitive rest for an undefined period of time until symptoms subside, followed by a sequence of physical and cognitive tests before being cleared to return to their daily activities (Choe, 2016). This separate evaluation of cognitive and motor domains is of significant concern: the integration of cognition and motor action, known as cognitive-motor integration (CMI), is essential to successful interaction with our environments. This interaction is contingent on our brain's ability to integrate sensory and motor information in order to execute an efficient motor plan - in other words, we often need to be able to think and move at the same time. The performance of tasks requiring $\mathrm{CMI}$ is dependent on intact connections between frontal, parietal, and subcortical brain regions (Gorbet \& Sergio, 2009; Gorbet \& Staines, 2011; Gorbet \& Sergio, 2016). Previous research has shown that following concussion, the integrity of these networks may be compromised, resulting in an impaired ability to integrate rules into coordinated motor tasks (Brown et al., 2015; Cook et al., 2020; Dalecki et al., 2016; Dalecki, Gorbet, Macpherson, et al., 2019; Hurtubise et al., 2016). However, patients are often cleared to return to their activities before CMI abilities have recovered (Brown et al., 2015; Hurtubise et al., 2016). Sex-related 
differences have also been observed in the brain networks which control CMI (Gorbet \& Sergio, 2007), and sex-related differences have been observed more generally in tasks requiring eyehand coordination (Gorbet et al., 2010; Gorbet \& Staines, 2011). Lastly, an increasing body of research is providing evidence for sex-related differences in concussion rates, symptoms, and recovery trajectory (Barnes et al., 1998; Black et al., 2016; Covassin et al., 2003, 2007, 2016; Gallagher et al., 2018; Ledoux et al., 2019; Merritt et al., 2019). Further, the vast majority of this work has been in the realm of sport-related concussion in youth and young adults (Berz et al., 2013; Frommer et al., 2011; Ono et al., 2015). Examining the utility of a concussion management program on skill recovery, and sex-related differences in this recovery, would be useful in order to fill the knowledge gap around concussion management for a broader demographic sample.

Ensuring that skilled performance has recovered following a concussive injury is important in that it will decrease vulnerability to another injury, and return an individual to their previous quality of daily life. The main aim of the present study was, therefore, to examine the recovery of CMI performance in concussed working-aged adults and adults with a history of concussion compared with healthy no-history controls during the course of a post-concussion management program. A secondary aim of this study was to examine whether sex- and agerelated differences were related to CMI performance over the duration of the program. As an exploratory aspect to the study, the relationship between CMI performance and symptom recovery was examined.

\section{Methods}

Participants

Fifteen adults with an acute concussion (mean age $27.33 \pm 6.94 ; 10$ females), 11 adults with a history of concussion (mean age $28.27 \pm 7.30 ; 7$ females), and 17 healthy controls (mean age $23.24 \pm 4.01 ; 8$ females, 1 unidentified) participated in this study. We obtained the information about concussion history and demographic data from all participants by our own and established questionnaires (SCAT5, Appendix A). Characteristics of participants detailing concussion history and demographic data are summarized in Table 1. All participants were recruited from the community. Participants were included in the acute concussion group if they were symptomatic or were between 10- and 125-days post-injury, and all acute concussions were 
physician diagnosed. This reference range was selected because of the timeframe during which symptom resolution is expected to occur in most individuals suffering from concussion, while also attempting to capture individuals with lingering symptoms. Individuals were included in the concussion history group if they reported a history of concussion of greater than 125 days prior to beginning the active recovery program and were asymptomatic. Participants were included in the healthy group if they reported no history of concussion. All individuals with cerebrovascular or cardiovascular disease, chronic headache, diabetes, cancer and clinical depression were excluded. Delivery of concussion management therapies and CMI testing took place at York University in Toronto, Ontario, Canada, between 2018 and 2019. The study protocol was approved by York University's Ethics Review Board and conformed to the standards of the Canadian Tri-Council Research Ethics guidelines. Participants signed written informed consent forms before participating in this study.

\section{Concussion management program}

Participants attended 3 sessions: 2 treatment sessions and a follow-up data session over the course of 6 weeks ( 1 session/2 weeks) (Figure 1). To test for concussion symptomatology, participants completed a health questionnaire in addition to a self-administered form (the SCAT5 Symptom Evaluation) and were administered the SCAT5 by a researcher. To test cognitivemotor abilities, participants were tested on two visuomotor transformation tasks, executed using the Brain Dysfunction Indicator (BrDI $\left.{ }^{\mathrm{TM}}\right)$. Participants were then provided osteopathic manual treatment (OMT), a form of manual therapy which has been shown to be effective in concussion management (Baltazar et al., 2020; Guernsey et al., 2016; Yao et al., 2020). Within a treatment session, participants were retested on the visuomotor tasks after manual therapy. During the final session, the same data collection procedures were followed, without the OMT.

\section{Procedures}

Participants performed two visuomotor transformation tasks that required sliding the index finger of the dominant hand directly along an ASUS touchscreen tablet (Asus Transformer Book T100TAF) or on an externally connected USB touchpad (Keytec ${ }^{\mathrm{TM}}$, TycoTouch, USA) situated perpendicular (in the horizontal plane) to the ASUS screen. The standard (direct) condition was the standard visuomotor mapping task, where the spatial location of the viewed 
target and the required movement were in alignment (hand movements were made on the ASUS tablet directly to the peripheral target) (Figure 2). The CMI condition was a non-standard visuomotor task that included two levels of decoupling. Participants were instructed to maintain their eye focus on the vertically oriented tablet while manipulating a cursor on the horizontally oriented Keytec ${ }^{\mathrm{TM}}$ touchpad. The feedback for this task was also rotated $180^{\circ}$ : for instance, to move the cursor to the left, the participant was required to slide their finger to the right.

The central yellow target appeared in the center of the screen for each trial and had a diameter of $7.5 \mathrm{~mm}$. Prior to initiation of the trial, the participant was instructed to place their finger in the center of the yellow target. After a delay period of $2000 \mathrm{~ms}$, a red peripheral target was presented $55 \mathrm{~mm}$ away from the center (up, down, left, right). This served as the 'Go' signal for the participant to slide their finger along the screen directly to the presented peripheral target. After reaching the peripheral target and remaining there for $500 \mathrm{~ms}$, the peripheral target disappeared. This served as the signal for the end of the trial. Following a delay of $2000 \mathrm{~ms}$, the central target reappeared, signaling the participant to return to the center for the next trial. The same sequence was followed for the CMI condition, but participants were sliding their fingers on the horizontal touchpad in the opposite direction of the presented target. Each participant completed 20 trials per condition ( 5 trials to each peripheral target), prior to and following manual therapy. Trials to the peripheral targets and condition order were presented in a randomized order. In both conditions, participants were instructed to move as quickly and as accurately as possible. Examples of full hand movement trajectories in healthy and acutely concussed participants in the CMI condition are plotted in Figure 3.

\section{Data processing and analysis}

Kinematic measures, including timing, finger position and error data were recorded for each trial and converted into a binary readable format using a custom written $\mathrm{C}++$ application. Unsuccessful trials were detected by the data collection software and resulted in trial termination if the finger left the home target too early $(<2000 \mathrm{~ms})$, reaction time $(\mathrm{RT})$ was too short $(<150$ $\mathrm{ms}$ ), RT was too long (>8000 ms), or movement time was too long (>10000 ms). Trials in which the first ballistic movement exited the boundaries of the center target in the wrong direction (greater than $45^{\circ}$ from a straight line to target) were coded as direction reversal errors, and analyzed as separate variables from the correct trials. A custom-written analysis program (using 
Matlab, Mathworks, Inc., USA) was used to generate a computerized velocity profile of each trial's movement, with movement onset and end being recorded at $10 \%$ peak velocity. These profiles were then verified by visual inspection, and corrections were performed when necessary. The scored data was processed to compute 7 different movement timing and execution outcome measures.

\section{Dependent measures}

The dependent variables of interest were reaction time, movement time, path length, peak velocity, the percent of trials resulting in a direction reversal, and movement accuracy and precision. The different variables are described in detail in the following section.

Movement timing variables. Reaction time (RT) was calculated as the time interval (milliseconds; ms) between the central target disappearance and movement onset. Movement time (MT) was calculated as the time in ms between movement onset and offset, and is composed of total movement (MTf, full movement offset) as well as ballistic movement (MTb, initial movement offset). If no corrective movements were made, ballistic movements were equivalent to full movement trajectories. To compare movement timing differences in the standard and CMI conditions, performance in the standard condition was subtracted from performance in the CMI condition $(\Delta \mathrm{RT} ; \Delta \mathrm{MT}$, calculated using MTf) at each time point. Peak velocity (PV) was the maximum velocity in $\mathrm{mm} / \mathrm{ms}$ obtained for each trial.

Pathlength. The ballistic pathlength (PLb) was recorded as the distance in mm between the starting position and the endpoint of the first ballistic movement (i.e., distance covered during the ballistic movement time). Full pathlength (PLf) was measured as the distance between start to final end location of the entire movement. Movements comprised of curves or deviations from a straight path between the central and peripheral target would thus result in a longer pathlength.

Endpoint analysis. Absolute error (AE), the end-point accuracy, was the average distance from the individual movement endpoints to the actual target location, in $\mathrm{mm}$. Variable error (VE), the end-point precision, describes the distance between the individual movement endpoints $(\sigma 2)$ from their mean movement, measured in $\mathrm{mm}$. 
Direction reversals. Direction reversals were only applicable in the CMI condition. They were calculated as the percentage of total trials that constituted a deviation of greater than $\pm 45^{\circ}$ from a straight line between the center of the central and peripheral targets (\%DRs).

\section{Statistical analysis}

Our statistical approach was to perform univariate and bivariate analyses in order to assess differences between groups and to look for possible significant differences in the dependent variables as a function of concussion history and sex, followed by multivariate analyses to examine the relationship between exposure variables of interest (age, concussion history, and sex) and the dependent variables, while controlling for the effect of these variables in the model.

Trials containing outcome measures $>2$ standard deviations (SDs) away from the mean for a given condition in a given participant were considered outliers and removed before statistical analysis. All remaining data were checked for normal distribution and sphericity (Mauchly's test), and were Greenhouse-Geisser corrected where necessary. Statistical analyses were performed using SPSS statistical software (IBM Inc.). Statistical significance levels were set to $\alpha \leq 0.05$.

Descriptive statistics were used to summarize subjects' characteristics. A frequency distribution was conducted on group (acute concussion, concussion history, healthy), sex, and recovery program completion. The mean and standard deviation of age was also determined. The normal distribution of all dependent variables was assessed, and the mean and standard deviation of each measure of visuomotor performance was determined.

Visuomotor task performance variables. For all dependent measures (RT, MTf, MTb, $\Delta \mathrm{RT}$, $\triangle \mathrm{MT}, \mathrm{PV}, \mathrm{PLf}, \mathrm{PLb}, \mathrm{AE}, \mathrm{VE}, \% \mathrm{DRs}$ ) in both conditions, effects of group (acute concussion, concussion history, healthy) and sex were analyzed separately using a repeated-measures mixed ANOVA, to test for any concussion- or sex-related behavioural differences. When there were significant main or interaction effects, pair-wise comparisons were used. Findings in either sex or concussion history were used to develop the models for linear regression. Note that for this study we constrained our analyses to a comparison of the behavioural data collected at the 
beginning of each treatment session. That is, the repeated measures analysis compared data across treatment sessions, rather than pre-post analysis within sessions.

Relationship between visuomotor task performance and participant characteristics. Blocked hierarchical linear regression analysis was used to determine if any of the independent variables can predict improvements in visuomotor performance. To investigate whether possible visuomotor performance deficits were related to concussion history (number of concussions), sex, or age over the course of an active recovery program, we correlated the dependent main variables of the direct condition and the CMI condition with these factors using a linear regression analysis. A 'pre-post' score was used in the linear regression, in which the final dependent measure score was subtracted from the initial score in order to measure changes in visuomotor performance prior to and following the program. A larger difference is indicative of improved performance. Individuals who did not complete the recovery program were excluded from this analysis.

Relationship between CMI performance and concussion symptom recovery. The relationship between CMI performance and number of concussion symptoms and severity (assessed using the SCAT5) was analyzed using Pearson's correlation.

\section{Results}

Table 1 presents selected characteristics of the participants of this study. Of the 43 participants, 17 were healthy (40\%), 11 were asymptomatic with a history of concussion (26\%), and 15 were acutely concussed (35\%). The total sample had a somewhat higher percentage of females (58\%), and the mean age was 25.95 . The median age of participants was 23 , ranging from 18 to 40 years old.

\section{Group comparisons}

The analysis using group as a main effect revealed significant differences in the CMI condition across the sessions for $\operatorname{MTb}(F=12.04, d f=2, p=0.000), \operatorname{MTf}(F=8.55, d f=2, p=$ $0.003)$ and $\mathrm{PV}(\mathrm{F}=9.84, \mathrm{df}=2, \mathrm{p}=0.001)$. Specifically, ballistic and full movement times decreased across the sessions, and peak velocity increased across the sessions in all groups. No 
significant differences were found for reaction time (RT) in either condition. The ANOVA also revealed a significant main effect of session number and a significant session number $\mathrm{X}$ group interaction for $\mathrm{AE}$ (endpoint accuracy) in the direct condition $(\mathrm{F}=3.18, \mathrm{df}=2, \mathrm{p}=0.05 ; \mathrm{F}(4,54)$ $=3.278, \mathrm{p}=0.018$ respectively), and a significant effect of session number in the CMI condition $(\mathrm{F}=4.92, \mathrm{DF}=2, \mathrm{p}=0.001)$. In the direct condition, there was an increase in $\mathrm{AE}$ across the sessions, and a significant difference was identified between the history and concussion groups. The concussion group had consistently larger AE scores across the 3 sessions compared to the history group ( $0.61 \mathrm{~mm}, \mathrm{p}=0.033$ ). In the $\mathrm{CMI}$ condition, there was a significant increase in $\mathrm{AE}$ across the sessions. However, since participants remained within the target, the increases in $\mathrm{AE}$ reflect participants ending their movements once directly inside the target, rather than aiming for the target center (from which accuracy was measured), rather than a worsening performance. A significant main effect of session number and a significant session number $\mathrm{X}$ group interaction was found for \%DRs in the CMI condition. Overall, a significant decrease in the \%DRs was identified across the sessions $(\mathrm{F}=3.84, \mathrm{df}=2, \mathrm{p}=0.027)$, though the interaction effect revealed that the observed decrease was driven by the concussion group $(F(4,60)=2.66, p=0.041)$. The healthy and history group maintained similar \%DRs in the CMI condition across the sessions, while the concussion group experienced a significant decrease in the percentage of reversal trials where they started off in the wrong direction, by the final session $(p=0.041)$. Despite the decrease, the concussion group still performed more \%DRs by the end of the program (Figure 4). Pair-wise comparisons revealed that the concussion group had an average $9.9 \%$ more DRs compared to the healthy group across the 3 sessions $(p=0.005)$, and an average $9.1 \%$ more DRs compared to the history group across the 3 sessions $(p=0.015)$.

To compare performance differences between the direct and CMI conditions, a difference score was calculated for the performance timing variables (CMI - Direct condition, calculated for all 3 sessions). The repeated-measures ANOVA on $\triangle \mathrm{MT}$ revealed a significant effect of session number $(\mathrm{F}=4.63, \mathrm{df}=2, \mathrm{p}=0.031)$, with a main effect of session number $\mathrm{X}$ group $(\mathrm{F}(2.49,33.59)=3.28, \mathrm{p}=0.04)$. The session number $\mathrm{X}$ group interaction revealed that decreases in $\triangle \mathrm{MT}$ were driven by the concussion group: the healthy and history groups maintained a similar $\triangle \mathrm{MT}$ throughout the recovery program, while the concussion group experienced a decrease in $\Delta \mathrm{MT}$ by the final session (Figure 5). 


\section{Sex-related comparisons}

The analysis using sex as a main effect revealed significant differences in the CMI condition across the sessions for $\operatorname{MTb}(\mathrm{F}=10.81, \mathrm{df}=2, \mathrm{p}=0.000), \operatorname{MTf}(\mathrm{F}=6.74, \mathrm{df}=1.2, \mathrm{p}=$ $0.01)$, and PV $(\mathrm{F}=9.1, \mathrm{df}=1.41, \mathrm{p}=0.002)$. In both sexes, a similar decrease in these variables was observed across all 3 sessions. The ANOVA also revealed a significant effect of session number for $\mathrm{AE}$ in the direct condition $(\mathrm{F}=4.26, \mathrm{df}=2, \mathrm{p}=0.019)$ and in the $\mathrm{CMI}$ condition $(\mathrm{F}=$ 4.73, $\mathrm{df}=2, \mathrm{p}=0.012$ ). A significant increase in AE across the sessions occurred in both conditions. Again, this likely indicates participants learning to stop immediately upon entering the target zone, rather than a worsening of performance. No interaction effects were observed between session number $\mathrm{X}$ sex.

Results of the ANOVAs revealed that session number (e.g., time) was the greatest factor affecting visuomotor performance, although group differences in concussion history emerged. For this reason, concussion history was chosen as a factor to include in the linear regression analysis. Though no significant differences were found by sex, sex differences have been identified in visuomotor performance (Gorbet et al., 2010; Gorbet \& Sergio, 2007; Rogojin et al., 2019); this factor was accordingly included in the regression analysis. Age has likewise been associated with recovery from concussion (Dalecki, Gorbet, \& Sergio, 2019), and was also included in the analysis. Despite a lack of significance in some of the measures of visuomotor performance, the regression was run on all dependent variables in both conditions, as visuomotor performance has not yet been investigated in both acute concussion and concussion history simultaneously by our group thus far. This was done by comparing performance in the $1^{\text {st }}$ and $3^{\text {rd }}$ sessions (pre-post score $=$ performance in session 1 - performance in session 3 ) for most dependent variables. For PV only, the pre-post score was calculated by subtracting performance in the $1^{\text {st }}$ session from performance in the $3^{\text {rd }}$ session (performance in session 3 -performance in session 1), as a larger PV in session 3 would indicate improved performance.

\section{Multivariate regression analyses}

In order to tease apart the relative effects of different factors that may contribute to performance recovery, a multivariate hierarchical linear regression resulted in a three-variable model of age, sex, and prior concussion as statistically significant predictors of visuomotor performance. Results of the final analyses on measures of visuomotor performance (taken as the 
difference in performance between session 1 and 3) are presented in Table 2. A greater difference represents an improvement in visuomotor performance in the $3^{\text {rd }}$ session compared to the first.

Movement timing variables. RT in the CMI condition was directly associated with concussion history (Figure 6). Individuals with 2 concussions experienced an improvement in RT compared to the healthy group, after adjusting for age and $\operatorname{sex}\left(\beta=127.64, p=0.047, R^{2}=0.117\right)$. MTb improvement in the standard condition was directly associated with group. Individuals who had experienced 2 concussions experienced a greater change in visuomotor performance compared to the healthy group, after adjusting for age and $\operatorname{sex}\left(\beta=391.51, \mathrm{p}=0.013, \mathrm{R}^{2}=0.269\right)$. In the CMI condition, MTb performance was associated with age and sex $\left(\mathrm{R}^{2}=0.329\right)$. Males experienced less improvement in MTb compared to females ( $\beta=-118.95, p=0.017$; Figure 7), and there was an increased improvement in visuomotor performance with every year aged $(\beta=11.98, p=$ 0.003). There was a significant association between MTf and concussion history in the standard condition: individuals who had sustained 2 concussions experienced a greater improvement in MTf compared to healthy controls, after adjusting for age and sex $\left(\beta=390.28, p=0.025, R^{2}=\right.$ 0.251). In the CMI condition, a significant association between age and sex was found after adjusting for concussion history $\left(\mathrm{R}^{2}=0.166\right)$. Male full movement times improved to a lesser extent than female full movement times $(\beta=-305.41, p=0.050$; Figure 7$)$, while there was a significant improvement in visuomotor performance with every year aged $(\beta=24.07, p=0.049)$. A significant association between age, concussion history, and skilled performance was found for PV. In the standard condition, individuals who had sustained 2 concussions experienced a greater improvement in PV compared to healthy controls, after adjusting for sex and age ( $\beta=$ 33.46, $\mathrm{p}=0.013, \mathrm{R}^{2}=0.212$; Figure 8 ). In the CMI condition, participants' improvements in PV increased with every year aged ( $\left.B=1.28, p=0.035, R^{2}=0.243\right)$. A trend was also observed between PV and number of concussions: individuals who had sustained 2 concussions experienced a greater improvement in PV compared to healthy controls $\left(\beta=22.64, \mathrm{p}=0.062, \mathrm{R}^{2}\right.$ $=0.243)$.

Path length. Concussion history was associated with PLf in the direct condition. Participants who sustained 3 or more concussions improved to a lesser extent than healthy controls ( $\beta=-$ 
4.923, $\mathrm{p}=0.047, \mathrm{R}^{2}=0.273$ ). There was an association between PLb and concussion history in the direct condition. After adjusting for age and sex, a trend was observed in which individuals who had sustained 3 or more concussions experienced smaller improvements compared to healthy controls $\left(\beta=-2.88, p=0.064, R^{2}=0.199\right)$.

Absolute error. Absolute error was directly associated with group. Participants who had sustained 3 or more concussions experienced a smaller improvement in the direct condition compared to healthy controls, after adjusting for age and sex $\left(B=-1.885, p=0.029, R^{2}=0.272\right)$. In the CMI condition, individuals who had sustained 2 concussions had a smaller change in visuomotor performance compared to healthy controls ( $\left.\beta=-4.599, \mathrm{p}=0.017, \mathrm{R}^{2}=0.216\right)$, and a trend was observed with sex: male improvements were smaller than female improvements ( $\beta=-$ $2.159, \mathrm{p}=0.072, \mathrm{R}^{2}=0.216$ ).

Variable error. No relationship was found between concussion history, age, or sex and VE performance.

Direction reversals. The \%DRs in the CMI condition was directly related to age. Individuals experienced a greater change in \%DRs every year aged, after adjusting for concussion history and sex $\left(B=0.615, p=0.022, R^{2}=0.209\right)$.

\section{Pearson correlations}

Results of the Pearson correlation indicated that there was a significant positive correlation between CMI performance deficits (\%DRs) and number of concussion symptoms prior to beginning the recovery program $(r=0.624, \mathrm{~N}=17, \mathrm{p}=0.007$; Figure 9). Following the recovery program, this relationship was no longer significant $(p=0.190)$. Similarly, a significant positive correlation was identified between CMI performance deficits (\%DRs) and severity of concussion symptoms prior to beginning the recovery program $(\mathrm{r}=0.576, \mathrm{~N}=17, \mathrm{p}=0.015$; Figure 10). Again, the relationship was no longer significant following completion of the program $(p=0.284)$. These findings suggest that in general, rule-based visuomotor skill recovers at the same rate as symptom recovery. 


\section{Discussion}

The present study sought to determine whether an active recovery program was associated with improved visuomotor performance in acutely concussed adults and adults with a history of concussion. A secondary aim of this study was to assess whether CMI deficits may be related to sex and age. Our findings indicate that performance on tasks requiring visuomotor integration can be improved over the course of a recovery program. We observed differences in performance on complex visuomotor tasks between acutely concussed individuals, individuals with a history of concussion, and healthy controls with no history of concussion. Specifically, participants who were acutely concussed had difficulty executing visually guided movements when there was a dissociation between the guiding visual information and the required motor action prior to the recovery program. These difficulties included deficits in movement planning and execution. However, following the program, participants with a history of concussion experienced larger improvements in performance on several visuomotor measures, as indicated by larger differences in performance between the first and final session, though there remained lingering deficits in some measures. Moreover, we observed sex-related differences in the performance of complex visuomotor tasks, with females outperforming males on the basis of movement timing variables. Finally, age moderated the performance of complex, rule-based tasks.

The results of the current study demonstrate that in both healthy individuals and individuals affected by concussion, performance of a rule-based movement task can be improved over time. Our findings also reveal that prior to a concussion recovery program, movement timing and accuracy in tasks requiring CMI are poor in those acutely affected by concussion. However, following the program, movements were faster and more accurate. These results complement findings from our previous studies with working aged healthy adults and adults atrisk for the development of Alzheimer's disease (AD), in which both groups experienced improvements in visuomotor performance (de Boer et al., 2018; Echlin et al., 2020). In combination with the findings from the current study, this suggests that both in the presence and absence of clinically altered brain function, visuomotor training may be beneficial for generalized improvement of functional ability. The ability to perform complex, rule-based tasks is crucial for many daily life and sport-related activities, and is impaired in several neurological conditions (Brown et al., 2015; Dalecki et al., 2016; Dalecki, Gorbet, Macpherson, et al., 2019; 
Rogojin et al., 2019). Our results support the notion that this important ability can be improved. It is likely that improvements in visuomotor performance occurred as a result of both time and treatment; thus, future work with a delayed-treatment control group will be investigating this possibility. The potential for improving motor functions requiring cognitive-motor integration is particularly important in individuals affected by brain dysfunction.

Our findings suggest the existence of visuomotor deficits in acutely concussed adults. As in our previous studies, the visuomotor deficits were evident when there was a dissociation between vision and action, and were observed in movement execution and movement accuracy (Brown et al., 2015; Dalecki et al., 2016). Prior to the recovery program, the CMI task appears to bring out behavioural deficits in acutely concussed participants. We suggest that these deficits may be due to disruptions in frontoparietal networks and in communication between brain regions responsible for the planning and execution of cognitive-motor integration. These deficits may likewise be due to altered integrity of frontoparietal-cerebellar white matter tracts - the cerebellum is heavily involved in CMI, given the need for online predictive movement feedback in the performance of these tasks (Gorbet \& Sergio, 2018, 2019). In fact, studies have reported frontoparietal network changes post-concussion. In particular, for networks that may be specifically related to CMI, studies with concussed individuals have found increased activation in parietal, frontal, and cerebellar regions when compared with pre-injury fMRI data (Gorbet \& Sergio, 2009, 2016; Granek \& Sergio, 2015; Hawkins et al., 2013; Sayegh et al., 2014; Slobounov et al., 2010; Tremblay et al., 2014). Moreover, anatomical studies have found altered diffusion characteristics within white matter tracts in concussed adolescents, in addition to younger and older adults with concussion history, in the pathways connecting frontal and parietal regions (Chamard et al., 2013; Hurtubise et al., 2020; Tremblay et al., 2014; Virji-Babul et al., 2013), as well as an association between white matter integrity differences along frontoparietalcerebellar white matter tracts and visuomotor performance in individuals affected by PCS (Chamard et al., 2013; Hurtubise et al., 2020). These data are in line with the current findings demonstrating impairment in tasks requiring CMI following concussion, suggesting that a crucial role is played by frontoparietal-cerebellar networks on tasks integrating thought and action (Granek \& Sergio, 2015; Hawkins et al., 2015; Sayegh et al., 2013, 2014). In the current study, the acutely concussed group improved over the course of the program while performance of other groups remained steady. This suggests that improvements in visuomotor performance may 
be related to the recovery of rule-based movement control brain networks. Future studies incorporating the use of functional magnetic resonance imaging (fMRI), electroencephalography (EEG), and functional near-infrared spectroscopy (fNIRS) would provide additional insight into the neural correlates of impaired performance, and into the underlying recovery of brain networks involved in the performance of complex, rule-based tasks following concussion.

Despite initial deficits compared to the healthy group, participants with a history of concussion experienced greater improvements in both standard tasks and tasks requiring CMI compared to healthy controls following the recovery program. Notably, improvements in movement timing and movement execution variables primarily occurred in individuals who had sustained 2 concussions, while participants who had sustained 1 or 3 or more concussions demonstrated either no improvements or decreased improvements compared to healthy controls. Our findings suggest an important role of number of concussions sustained in the ability to recover performance of complex visuomotor tasks. Multiple concussions have been associated with cognitive and motor deficits, psychiatric impairments, neurodegenerative diseases, and impaired recovery, though most of the literature investigates these effects in athletes (Covassin et al., 2013; Teel et al., 2017). Studies have reported that across the lifespan, prior concussion history is associated with less recovery in athletes compared to athletes sustaining their first concussion (Wojcik, 2014). Moreover, history of multiple concussions, generally defined as 2 or more concussions, is associated with altered balance and gait characteristics, in addition to prolonged neurocognitive and symptom recovery, as indicated by larger reaction times and lower memory performance (Brooks et al., 2014; Covassin et al., 2008; Howell et al., 2017; Iverson et al., 2004; Teel et al., 2017). Impairments associated with multiple concussion in athletes may be due to electrophysiological changes and disrupted communication between brain areas involved in the performance of these tasks. In fact, studies have demonstrated supressed electrophysiological activity in asymptomatic multiple-concussion athletes compared to healthy controls, after adjusting for time since the latest concussion (de Beaumont et al., 2007; Thériault et al., 2009). Similarly, functional connectivity in the anterior default mode network is significantly lower after sustaining multiple concussions compared to sustaining 1 concussion (Plourde et al., 2020). Though our data demonstrate that improved CMI performance occurs in those with a history of 2 concussions, prior studies do not differentiate between 2 and 3 or more concussions. Our findings support the notion that multiple concussions are impairing recovery, 
as evidenced by diminished visuomotor performance improvements in individuals who sustained 3 or more concussions compared to healthy controls, though we further distinguish that sustaining 1 concussion may result in comparable performance to healthy controls, while 2 concussions may allow the ability to recover performance deficits. Thus, there may be a 'doseresponse' relationship between the number of concussions and the ability to recover cognitivemotor function. More research is needed in the non-athlete population in order to further support this notion.

Sex-related differences and an effect of age were observed in this study. After concussion history and age were adjusted for, male movement times in the direct and CMI conditions improved to a lesser extent than female movement times. This is consistent with a previous study from our group demonstrating that males performed worse in a CMI task (Rogojin et al., 2019). Furthermore, previous studies have observed that both CMI and standard tasks evoke a notably more bilateral pattern of activity in premotor and parietal regions in women compared to men, though in CMI tasks, men have greater lateral sulcus activity than women (Gorbet \& Sergio, 2007; Sergio et al., 2020). Thus, differences in brain activation patterns may contribute to observed differences in the current study, though it is also likely that since males typically perform worse in visuomotor tasks, there is the potential for stronger recovery on CMI measures in females compared to males.

Finally, in the CMI condition, aging was associated with a greater improvement in performance. Though visuomotor performance declines are typically associated with aging (de Boer et al., 2018; Echlin et al., 2020; Rogojin et al., 2019), our study investigated performance in young to working-aged adults. Therefore, performance declines would not be expected to occur with age in this group. In younger age groups, adolescents demonstrate improved performance in CMI tasks compared to children (11-12 years old) and young children (8-10 years old) (Dalecki et al., 2016). Moreover, children and adolescents with concussion history perform worse than young adults with concussion history (Dalecki et al., 2016). Taken together, the evidence suggests that visuomotor performance follows an inverted U-shaped curve in aging, during which growing and aging brains are neurologically more fragile for executing CMI tasks. This may explain the mechanism underlying the improvement associated with age in the current study: younger participants' brain networks were more fragile than older ones. Thus, 
working-aged adults in this study may have stronger networks controlling the performance of complex, rule-based movements, and this may underlie the greater improvements seen with age. Sensorimotor integration is essential to the performance of complex, rule-based tasks. Performance on such tasks is impaired by concussion, and it is therefore essential to incorporate recovery of visuomotor skill performance in concussion management. The goal of the present study was to examine the recovery of CMI in concussed working-aged adults and adults with a history of concussion compared with healthy no-history controls during the course of a postconcussion management program. Prior to the program, individuals affected by concussion demonstrated impaired performance on complex visuomotor tasks. Over the course of the program, greater improvements were observed in participants with a history of 2 concussions compared to no-concussion-history healthy controls. Moreover, females demonstrated a stronger recovery of these skills compared to males, and a positive effect of age was observed. These data suggest that the number of concussions sustained affects the integrity of brain networks controlling skilled performance, and that the underlying brain networks that control cognitivemotor integration are different between males and females. These differences should be further investigated in a broader age range, as these results provide important factors to consider in concussion management. 


\section{Tables}

Table 1. Characteristics for 15 acutely concussed participants, 11 participants with a concussion history, and 17 participants with no history of concussion.

\begin{tabular}{|lllll|}
\hline Variables & & $\mathrm{N}(\%)$ & Mean (SD) & Median (Range) \\
\hline $\begin{array}{l}\text { Age (years) } \\
\text { Sex }\end{array}$ & & $25.95(6.137)$ & $23(18-40)$ \\
\hline & Female & $25(58.1)$ & \\
\hline & Male & $17(39.5)$ & \\
\hline Concussion history & Unreported & $1(2.31)$ & \\
& Healthy & $17(39.5)$ & \\
\hline & History & $11(25.6)$ & \\
\hline & Acute Concussion & $15(34.9)$ & \\
\hline
\end{tabular}

Table 2. Association between visuomotor performance and history of concussion, sex, and age. $(\mathrm{N}=33)$.

\begin{tabular}{|c|c|c|c|c|}
\hline & & & Model $1\left(R^{\wedge} 2=0.209\right)$ & \\
\hline \multirow[t]{6}{*}{ \% Direction Reversal, CMI Condition } & & Unstandardized B & $95 \% \mathrm{CI}$ & p value \\
\hline & Age & 0.615 & $0.092,1.138$ & 0.022 \\
\hline & 1 Concussion $^{0}$ & -2.995 & $-11.127,5.138$ & 0.46 \\
\hline & 2 Concussions $^{0}$ & 2.444 & $-8.078,12.966$ & 0.64 \\
\hline & $3+$ Concussions $^{\circ}$ & 3.414 & $-4.918,11.745$ & 0.411 \\
\hline & Male $\dagger$ & -4.197 & $-10.853,2.459$ & 0.209 \\
\hline \multirow[t]{7}{*}{ Reaction Time (ms), Direct Condition } & & & Model $1\left(R^{\wedge} 2=0.063\right)$ & \\
\hline & & Unstandardized B & $95 \%$ CI & p value \\
\hline & Age & 1.223 & $-4.717,7.163$ & 0.679 \\
\hline & 1 Concussion $^{\circ}$ & -10.907 & $-103.295,81.482$ & 0.812 \\
\hline & 2 Concussions $^{\circ}$ & -57.344 & $-176.880,62.191$ & 0.337 \\
\hline & $3+$ Concussions $^{\circ}$ & 19.979 & $-74.670,114.629$ & 0.671 \\
\hline & Male $\dagger$ & 25.059 & $-50.557,100.675$ & 0.506 \\
\hline \multirow[t]{7}{*}{ Reaction Time (ms), CMI Condition } & & & Model $1\left(R^{\wedge} 2=0.117\right)$ & \\
\hline & & Unstandardized B & $95 \%$ CI & p value \\
\hline & Age & -1.633 & $-7.872,4.607$ & 0.599 \\
\hline & 1 Concussion $^{\circ}$ & 33.561 & $-63.489,130.611$ & 0.488 \\
\hline & 2 Concussions $^{\circ}$ & 127.642 & $2.076,253.207$ & 0.047 \\
\hline & $3+$ Concussions $^{0}$ & 61.34 & $-38.085,160.764$ & 0.219 \\
\hline & Male $\dagger$ & -2.851 & $-82.282,76.580$ & 0.942 \\
\hline \multicolumn{2}{|l|}{ Full Movement Time (ms), Direct Condition } & & Model $1\left(R^{\wedge} 2=0.251\right)$ & \\
\hline
\end{tabular}




\begin{tabular}{|c|c|c|c|c|}
\hline & & Unstandardized B & $95 \%$ CI & p value \\
\hline & Age & 5.21 & $-11.612,22.031$ & 0.534 \\
\hline & 1 Concussion $^{\circ}$ & 10.571 & $-251.077,272.220$ & 0.935 \\
\hline & 2 Concussions $^{\circ}$ & 390.277 & $51.749,728.805$ & 0.025 \\
\hline & $3+$ Concussions $^{\circ}$ & -138.225 & $-406.276,129.826$ & 0.303 \\
\hline & Male $\dagger$ & -134.857 & $-349.004,79.290$ & 0.21 \\
\hline \multirow[t]{7}{*}{ Full Movement Time (ms), CMI Condition } & & & Model $1\left(R^{\wedge} 2=0.166\right)$ & \\
\hline & & Unstandardized B & $95 \% \mathrm{CI}$ & p value \\
\hline & Age & 24.071 & $0.060,48.083$ & 0.049 \\
\hline & 1 Concussion $^{\circ}$ & -85.1 & $-458.854,288.383$ & 0.647 \\
\hline & 2 Concussions $^{\circ}$ & -95.846 & $-579.069,387.378$ & 0.69 \\
\hline & 3+ Concussions ${ }^{\circ}$ & -53.434 & $-436.056,329.188$ & 0.779 \\
\hline & Male $\dagger$ & -305.414 & $-611.092,0.265$ & 0.05 \\
\hline \multicolumn{2}{|l|}{ Ballistic Movement Time (ms), Direct Condition } & & Model $1\left(R^{\wedge} 2=0.269\right)$ & \\
\hline & & Unstandardized B & $95 \% \mathrm{CI}$ & p value \\
\hline & Age & 4.884 & $-10.281,20.049$ & 0.518 \\
\hline & 1 Concussion $^{\circ}$ & -21.026 & $-256.908,214.856$ & 0.858 \\
\hline & 2 Concussions $^{\circ}$ & 391.507 & $86.316,696.698$ & 0.013 \\
\hline & $3+$ Concussions $^{\circ}$ & -101.984 & $-343.638,139.670$ & 0.398 \\
\hline & Male $\dagger$ & -122.994 & $-316.052,70.064$ & 0.205 \\
\hline \multicolumn{2}{|l|}{ Ballistic Movement Time (ms), CMI Condition } & & Model $1\left(R^{\wedge} 2=0.329\right)$ & \\
\hline & & Unstandardized B & $95 \%$ CI & p value \\
\hline & Age & 11.975 & $4.438,19.512$ & 0.003 \\
\hline & 1 Concussion $^{0}$ & 52.637 & $-64.595,169.869$ & 0.369 \\
\hline & 2 Concussions $^{\circ}$ & 39.144 & $-112.534,190.821$ & 0.604 \\
\hline & 3+ Concussions $^{\circ}$ & 32.158 & $-87.943,152.258$ & 0.59 \\
\hline & Male $\dagger$ & -118.954 & $-214.903,-23.005$ & 0.017 \\
\hline \multirow[t]{7}{*}{ Full Path Length (mm), Direct Condition } & & & Model $1\left(R^{\wedge} 2=0.273\right)$ & \\
\hline & & Unstandardized B & $95 \%$ CI & p value \\
\hline & Age & -0.149 & $-0.422,0.125$ & 0.273 \\
\hline & 1 Concussion $^{0}$ & -0.767 & $-5.065,3.530$ & 0.716 \\
\hline & 2 Concussions $^{\circ}$ & -0.223 & $-5.118,4.671$ & 0.926 \\
\hline & 3+ Concussions ${ }^{0}$ & -4.923 & $-9.776,-0.071$ & 0.047 \\
\hline & Male $\dagger$ & -1.002 & $-4.763,2.759$ & 0.587 \\
\hline \multirow[t]{7}{*}{ Full Path Length (mm), CMI Condition } & & & Model $1\left(R^{\wedge} 2=0.192\right)$ & \\
\hline & & Unstandardized B & $95 \% \mathrm{CI}$ & p value \\
\hline & Age & 1.417 & $-0.265,3.099$ & 0.095 \\
\hline & 1 Concussion $^{\circ}$ & -19.651 & $-47.977,8.674$ & 0.166 \\
\hline & 2 Concussions $^{\circ}$ & -23.167 & $-55.731,9.397$ & 0.156 \\
\hline & $3+$ Concussions $^{\circ}$ & -9.568 & $-37.644,18.509$ & 0.491 \\
\hline & Male $\dagger$ & -21.379 & $-44.420,1.662$ & 0.068 \\
\hline
\end{tabular}




\begin{tabular}{|c|c|c|c|c|}
\hline \multicolumn{2}{|l|}{ Ballistic Path Length (mm), Direct Condition } & \multirow[b]{2}{*}{ Unstandardized B } & \multirow{2}{*}{$\begin{array}{l}\text { Model } 1\left(R^{\wedge} 2=0.199\right) \\
95 \% \text { CI }\end{array}$} & \multirow[b]{2}{*}{ p value } \\
\hline & & & & \\
\hline & Age & -0.133 & $-0.324,0.059$ & 0.17 \\
\hline & 1 Concussion $^{\circ}$ & -0.677 & $-3.661,2.306$ & 0.648 \\
\hline & 2 Concussions ${ }^{0}$ & -0.023 & $-3.883,3.838$ & 0.991 \\
\hline & 3+ Concussions ${ }^{\circ}$ & -2.88 & $-5.937,0.176$ & 0.064 \\
\hline & Male $\dagger$ & -1.295 & $-3.737,1.147$ & 0.289 \\
\hline \multicolumn{2}{|l|}{ Ballistic Path Length (mm), CMI Condition } & & Model $1\left(R^{\wedge} 2=0.141\right)$ & \\
\hline & & Unstandardized B & $95 \% \mathrm{CI}$ & p value \\
\hline & Age & 1.177 & $-0.239,2.593$ & 0.1 \\
\hline & 1 Concussion $^{\circ}$ & -11.673 & $-33.694,10.347$ & 0.289 \\
\hline & 2 Concussions $^{\circ}$ & -18.234 & $-46.724,10.257$ & 0.203 \\
\hline & 3+ Concussions ${ }^{\mathbf{O}}$ & -8.332 & $-30.891,14.227$ & 0.459 \\
\hline & Male $\dagger$ & -14.531 & $-32.553,3.492$ & 0.111 \\
\hline \multicolumn{2}{|l|}{ Absolute Error (mm), Direct Condition } & & Model $1\left(R^{\wedge} 2=0.272\right)$ & \\
\hline & & Unstandardized B & $95 \% \mathrm{CI}$ & p value \\
\hline & Age & -0.076 & $-0.181,0.029$ & 0.152 \\
\hline & 1 Concussion $^{\circ}$ & -1.057 & $-2.693,0.579$ & 0.198 \\
\hline & 2 Concussions ${ }^{0}$ & 1.024 & $-1.092,3.140$ & 0.333 \\
\hline & $3+$ Concussions $^{\circ}$ & -1.885 & $-3.560,-0.209$ & 0.029 \\
\hline & Male $\dagger$ & -0.795 & $-2.133,0.544$ & 0.237 \\
\hline \multirow[t]{7}{*}{ Absolute Error (mm), CMI Condition } & & & Model $1\left(R^{\wedge} 2=0.216\right.$ & \\
\hline & & Unstandardized B & $95 \% \mathrm{CI}$ & p value \\
\hline & Age & 0.15 & $-0.035,0.336$ & 0.109 \\
\hline & 1 Concussion ${ }^{0}$ & -0.87 & $-3.754,2.014$ & 0.544 \\
\hline & 2 Concussions $^{0}$ & -4.599 & $-8.330,-0.867$ & 0.017 \\
\hline & $3+$ Concussions $^{\circ}$ & -0.736 & $-3.691,2.218$ & 0.616 \\
\hline & Male $\dagger$ & -2.159 & $-4.519,0.202$ & 0.072 \\
\hline \multirow[t]{7}{*}{ Variable Error (mm), Direct Condition } & & & Model $1\left(R^{\wedge} 2=0.111\right)$ & \\
\hline & & Unstandardized B & $95 \% \mathrm{CI}$ & p value \\
\hline & Age & 0.014 & $-0.018,0.047$ & 0.376 \\
\hline & 1 Concussion ${ }^{\circ}$ & 0.266 & $-0.240,0.771$ & 0.294 \\
\hline & 2 Concussions $^{0}$ & 0.163 & $-0.491,0.818$ & 0.616 \\
\hline & 3+ Concussions ${ }^{\circ}$ & 0.396 & $-0.122,0.914$ & 0.13 \\
\hline & Male $\dagger$ & -0.17 & $-0.584,0.244$ & 0.41 \\
\hline \multirow[t]{6}{*}{ Variable Error (mm), CMI Condition } & & & Model $1\left(R^{\wedge} 2=0.091\right)$ & \\
\hline & & Unstandardized B & $95 \% \mathrm{CI}$ & p value \\
\hline & Age & 0.024 & $-0.015,0.062$ & 0.221 \\
\hline & 1 Concussion $^{0}$ & 0.067 & $-0.531,0.665$ & 0.823 \\
\hline & 2 Concussions $^{\circ}$ & 0.297 & $-0.477,1.07$ & 0.442 \\
\hline & $3+$ Concussions $^{\circ}$ & -0.164 & $-0.776,0.449$ & 0.592 \\
\hline
\end{tabular}




\begin{tabular}{|c|c|c|c|c|}
\hline & Male $\dagger$ & -0.132 & $-0.621,0.358$ & 0.588 \\
\hline \multicolumn{2}{|l|}{ Peak Velocity $(\mathrm{mm} / \mathrm{ms})$, Direct Condition } & & Model $1\left(R^{\wedge} 2=0.212\right)$ & \\
\hline & & Unstandardized B & $95 \%$ CI & p value \\
\hline & Age & 0.116 & $-1.178,1.411$ & 0.856 \\
\hline & 1 Concussion $^{\circ}$ & 2.741 & $-17.4,22.881$ & 0.784 \\
\hline & 2 Concussions $^{0}$ & 33.46 & $7.402,59.519$ & 0.013 \\
\hline & $3+$ Concussions $^{\circ}$ & -6.584 & $-27.217,14.05$ & 0.522 \\
\hline & Male $\uparrow$ & -2.409 & $-18.894,14.075$ & 0.769 \\
\hline \multicolumn{2}{|l|}{ Peak Velocity $(\mathrm{mm} / \mathrm{ms})$, CMI Condition } & & Model $1\left(R^{\wedge} 2=0.243\right)$ & \\
\hline & & Unstandardized B & $95 \% \mathrm{CI}$ & p value \\
\hline & Age & 1.276 & $0.092,2.46$ & 0.035 \\
\hline & 1 Concussion $^{\circ}$ & 9.998 & $-8.415,28.41$ & 0.278 \\
\hline & 2 Concussions $^{\circ}$ & 22.641 & $-1.182,46.464$ & 0.062 \\
\hline & $3+$ Concussions $^{\circ}$ & 5.528 & $-13.335,24.391$ & 0.556 \\
\hline & Male $\dagger$ & 1.026 & $-14.044,16.096$ & 0.891 \\
\hline
\end{tabular}

Blocked hierarchical linear regression model 1 adjusts for age, history of concussion, and sex

${ }^{\circ}$ Reference category = healthy adults (no history of concussion).

$\dagger$ Males compared to females.

For each unit of the participants' characteristics there is on average an increase or a decrease in unstandardized B, representing the change in visuomotor performance scores (pre-recovery program score - post-recovery program score). A positive unstandardized B indicates a greater change in participants' scores (an improvement) and a negative unstandardized B indicates a smaller change in participants' scores. 


\section{Figures}

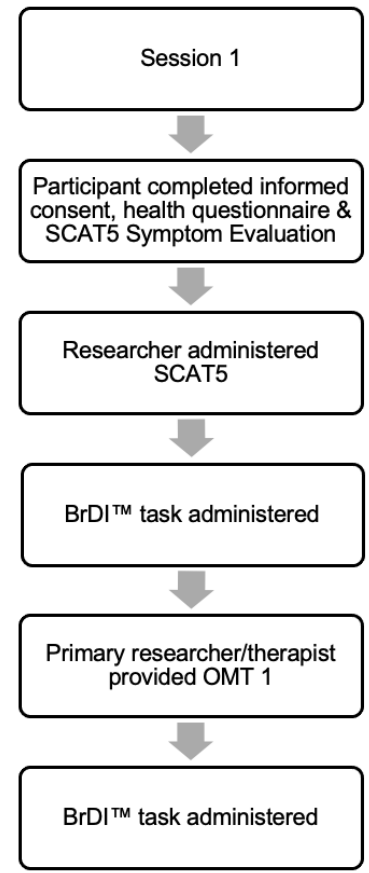

Baseline

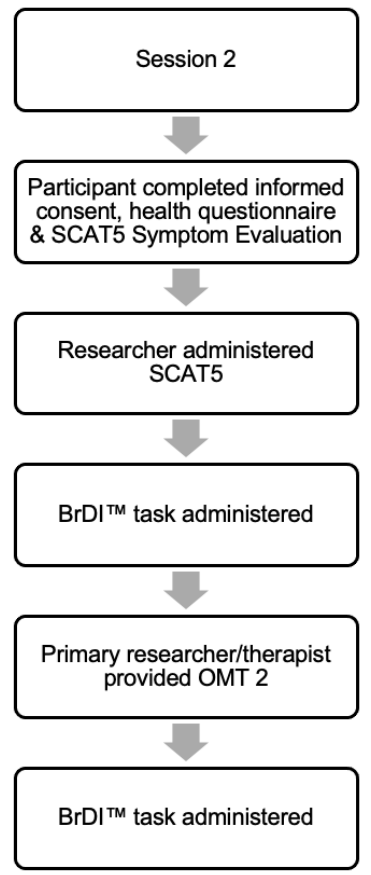

Mid-Study

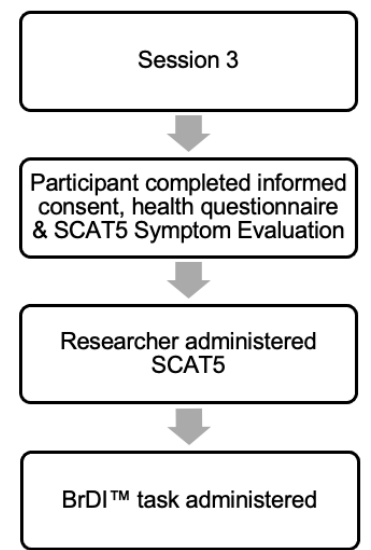

\section{Study Completion}

\begin{tabular}{|c|c|}
\hline OSTEOPATHIC MANUAL TREATMENT 1 & OSTEOPATHIC MANUAL TREATMENT 2 \\
\hline $\begin{array}{ll} & \mathrm{C} 0-\mathrm{C} 1, \mathrm{C} 1-\mathrm{C} 2, \mathrm{C} 1 / \mathrm{C} 2-\mathrm{C} 3 \\
& \text { compaction treatment } \\
\text { - } & \mathrm{SBS} \text { assessment \& decompaction } \\
\text { - } & \text { OM suture assessment \& } \\
\text { decompaction } \\
\text { - }\end{array}$ & 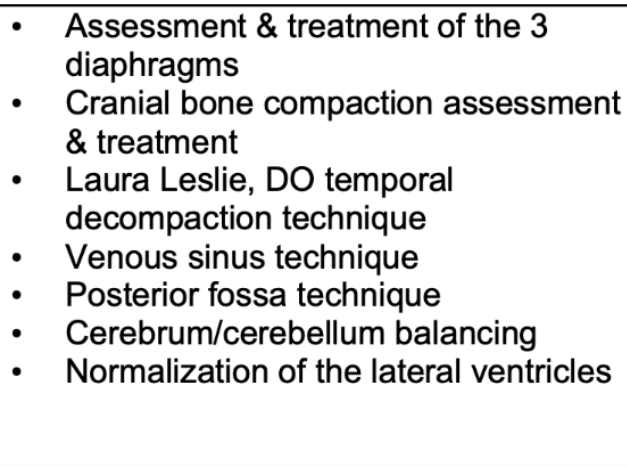 \\
\hline
\end{tabular}

Figure 1. Research procedure flow diagram and osteopathic techniques provided during this study. 


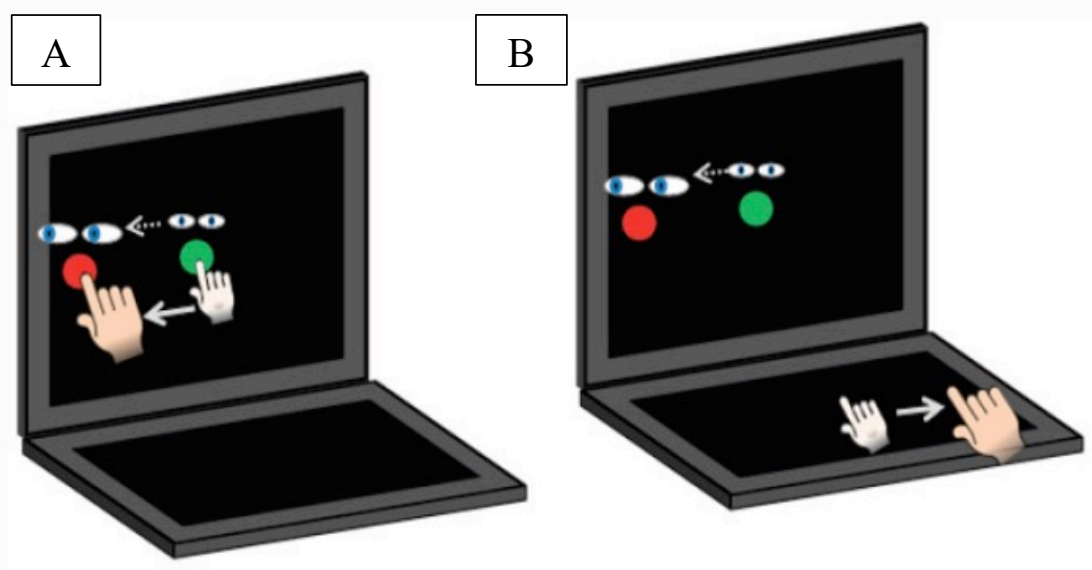

Figure 2. Illustration of the tablet-based BrDITM task. The green circle denotes the central home target in which all movements begin. A red target appears in one of 4 peripheral direction (top, bottom, left, or right of centre) after $2000 \mathrm{~ms}$ which serves as the 'Go' cue. (A) The standard (ST) condition, in which eye and arm movements are congruent (moved to the same peripheral target). (B) The non-standard cognitive-motor integration (CMI) condition, in which vision and movement are decoupled to a plane dissociation (eyes look at the vertical screen while hand moves along the horizontal screen), and visual feedback reversal (cursor movement $180^{\circ}$ rotated from hand motion). 

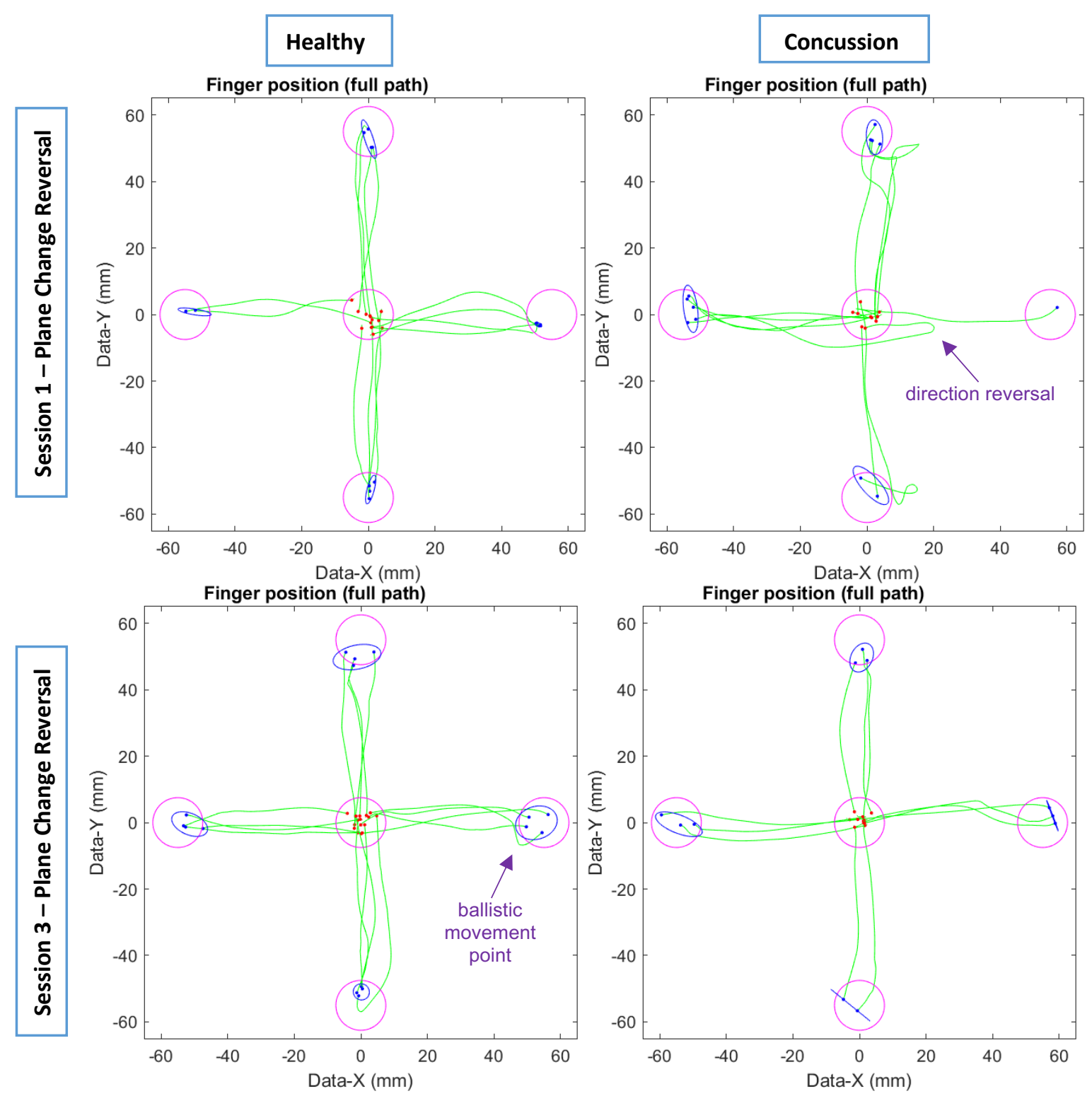

Figure 3. Examples of typical full hand movement trajectories for both healthy (left) and concussed (right) individuals, at start (top) and end (bottom) of the program. Trajectories begin at the central target (red dots) and move towards one of four peripheral targets, where each green line represents a single movement trajectory. Blue ellipses denote the $95 \%$ C.I. for the final end point of the finger movements (blue dots). Correct trials (green lines) are shown. Any target with less than 5 trajectories indicates error trials (not shown). Ballistic measures are taken from initial slowing point ("ballistic movement point", $<10 \%$ peak velocity). 


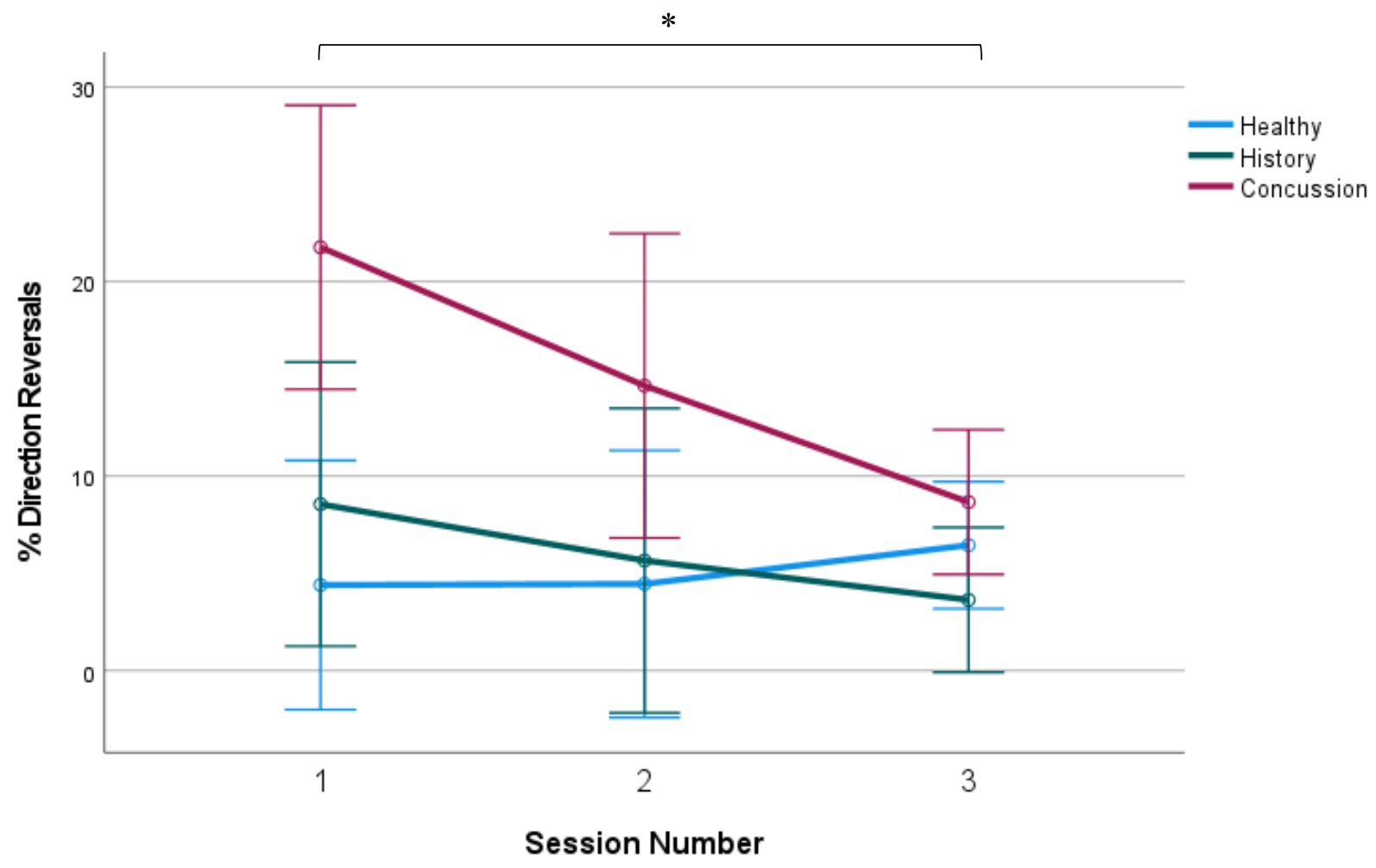

Error bars: $95 \% \mathrm{Cl}$

Figure 4. Percent of trials in the CMI condition resulting in direction reversals, by session and group. The percent of direction reversals is significantly greater between the concussion group and the healthy and history groups during session 1 . Following the program time, the concussion group experiences a significant improvement. *: $\mathrm{p}<0.05$ 


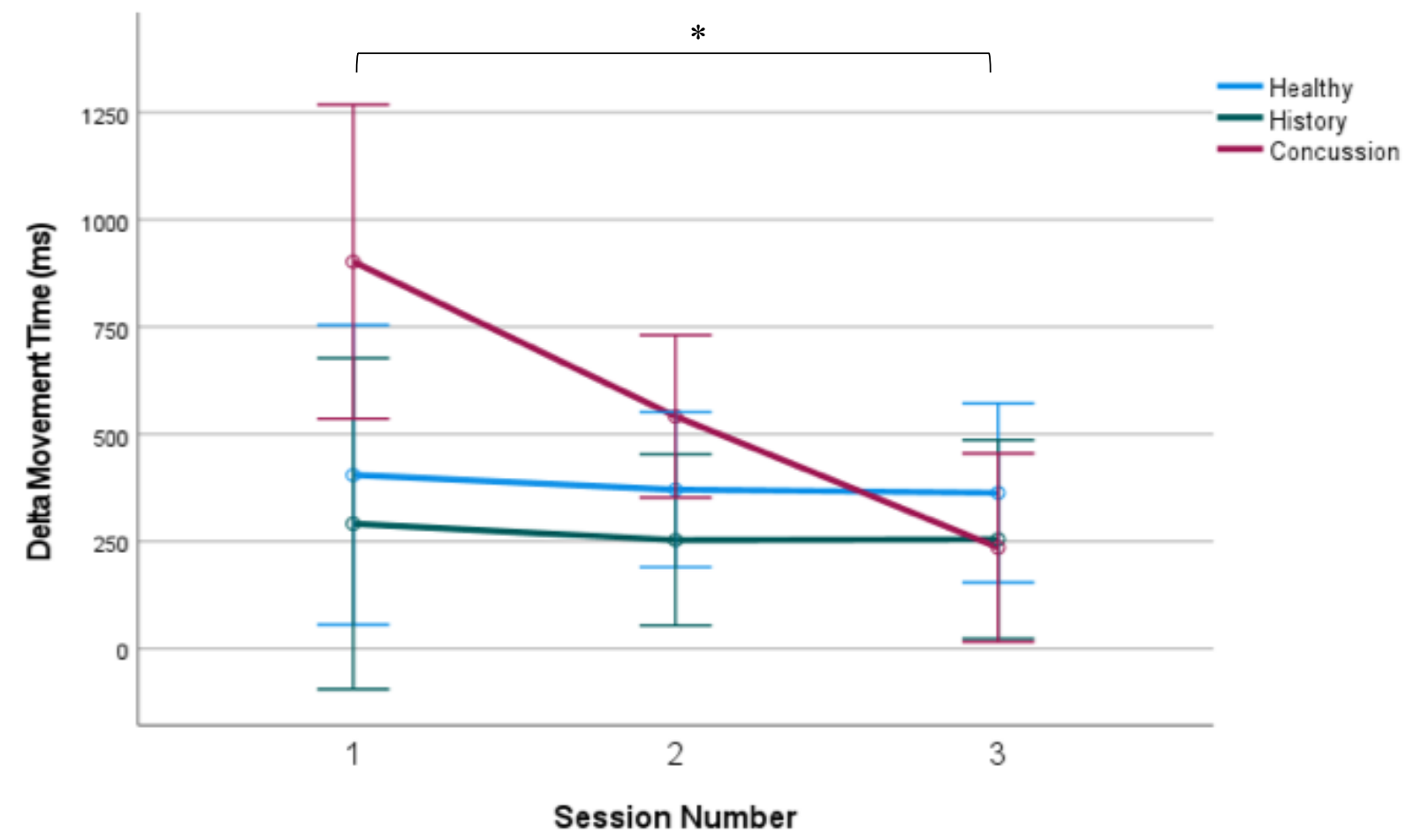

Error bars: $95 \% \mathrm{Cl}$

Figure 5. Difference in Movement Time in the CMI and Direct Condition, by session and group. The difference is significantly greater between the concussion group and the healthy and history groups during session 1 (delta MT). Following the program time, the concussion group experiences a significant improvement. $*$ : $\mathrm{p}<0.05$ 


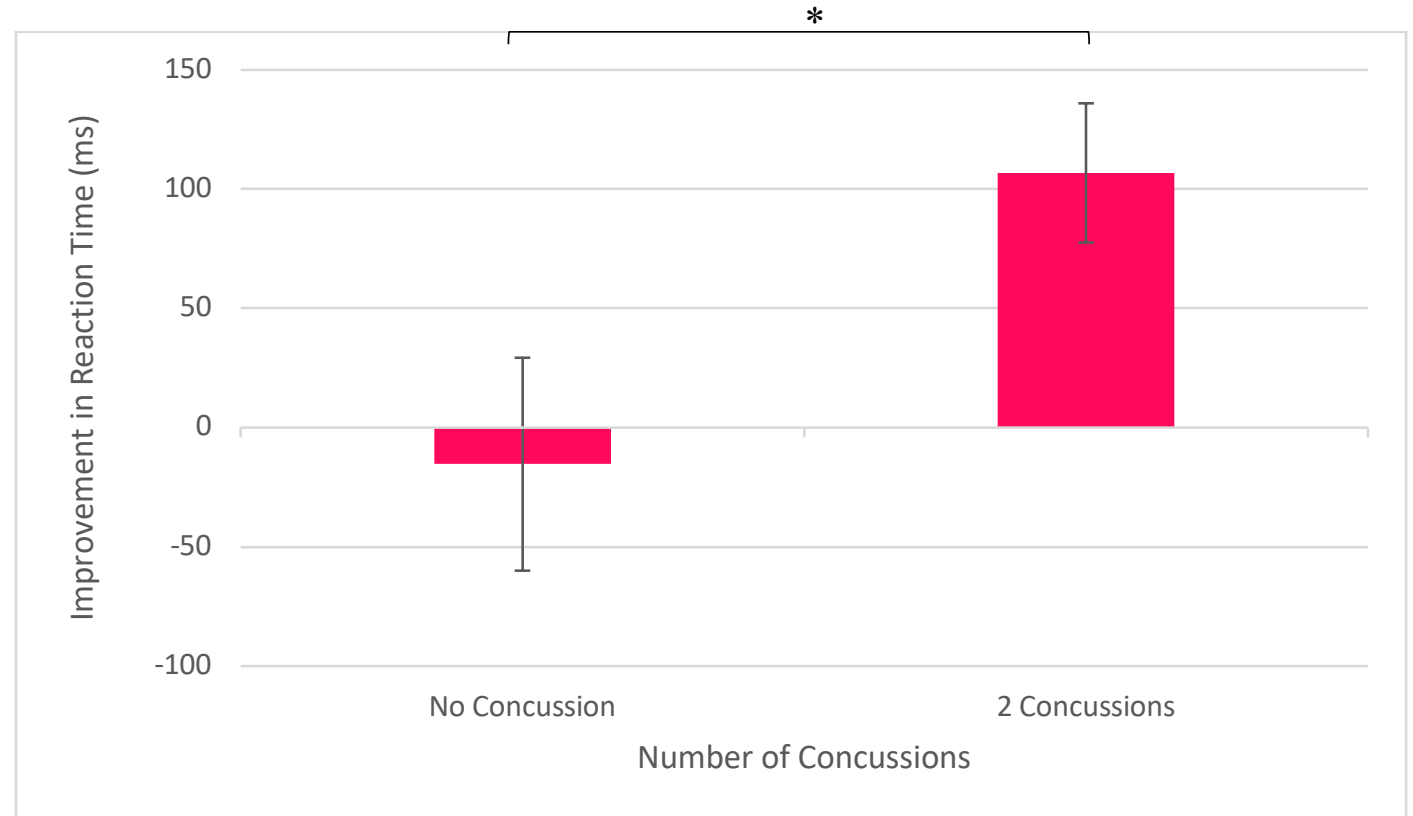

Figure 6. Mean improvement in reaction time over a concussion recovery program in the CMI condition, as a function of number of concussions. Participants with a history of 2 concussions improved their reaction times significantly more than healthy controls. *: $p<0.05$ 


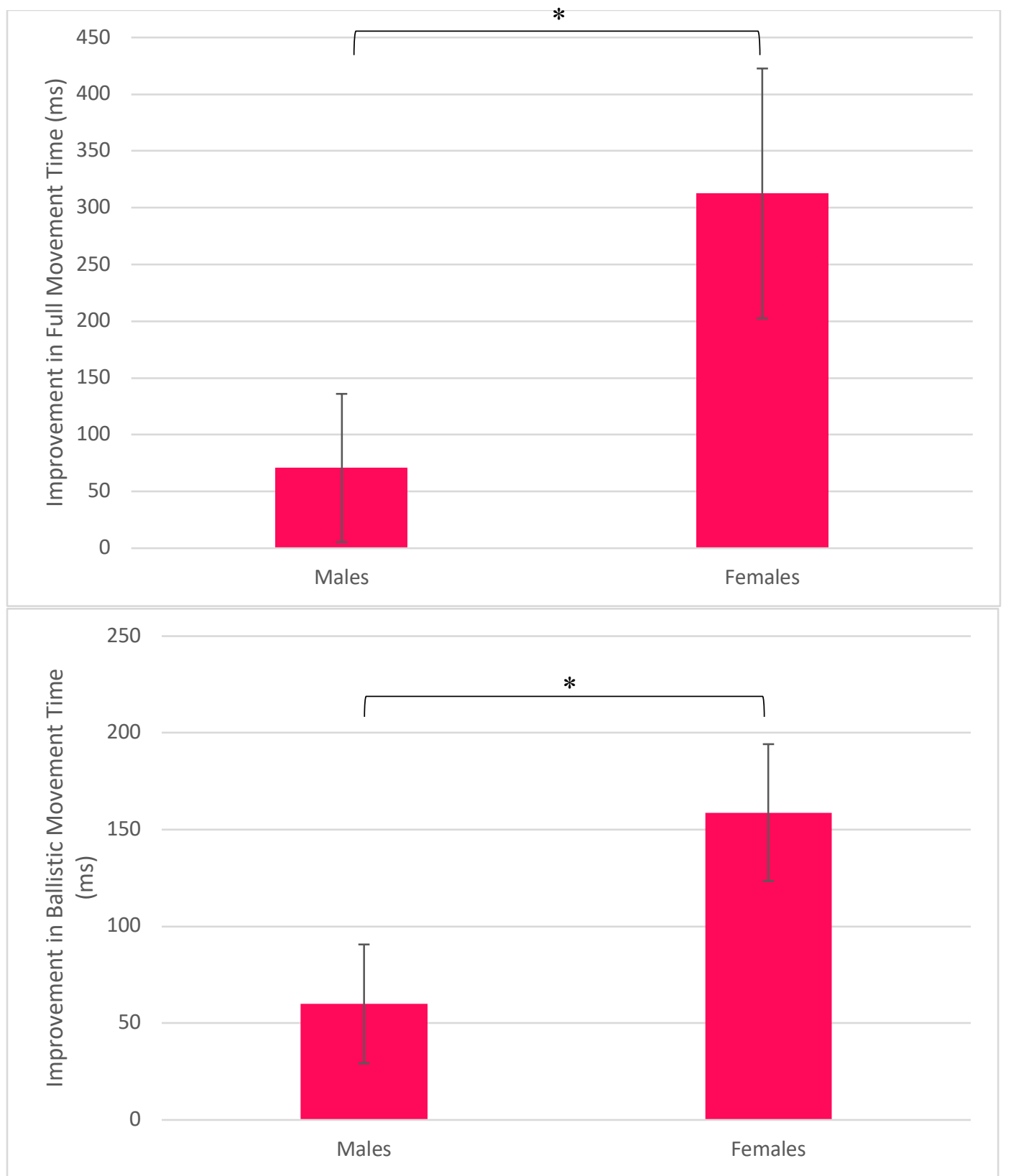

Figure 7. Mean improvements in full (top) and ballistic (bottom) movement time over a concussion recovery program in the CMI condition, as a function of sex. Females improved their full and ballistic movement times significantly more than males. ${ }^{*}: \mathrm{p}<0.05$ 


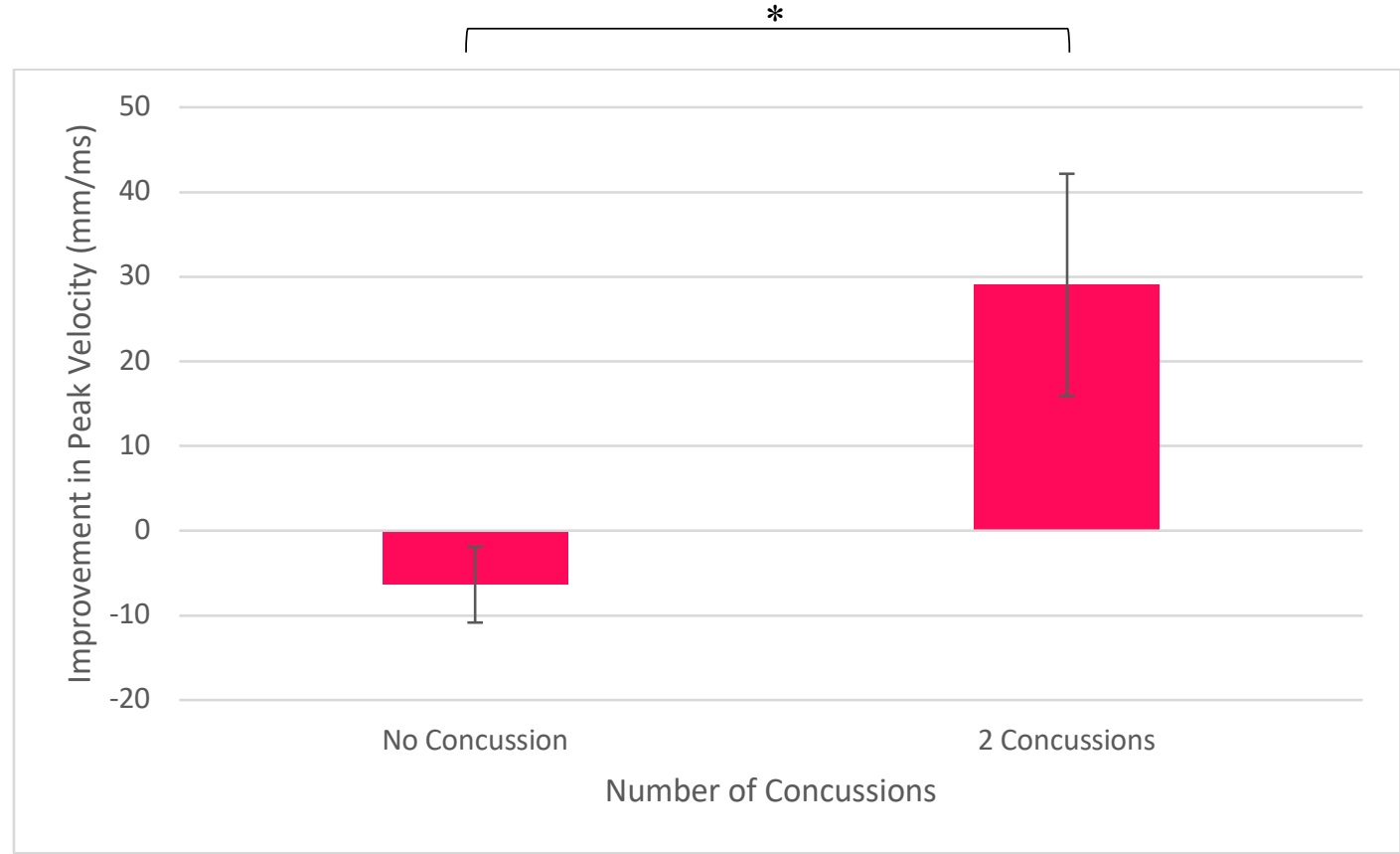

Figure 8. Mean improvement in peak velocity over a concussion recovery program in the Direct condition, as a function of number of concussions. Participants with a history of 2 concussions improved their peak velocities significantly more than healthy controls. *: $p<0.05$ 


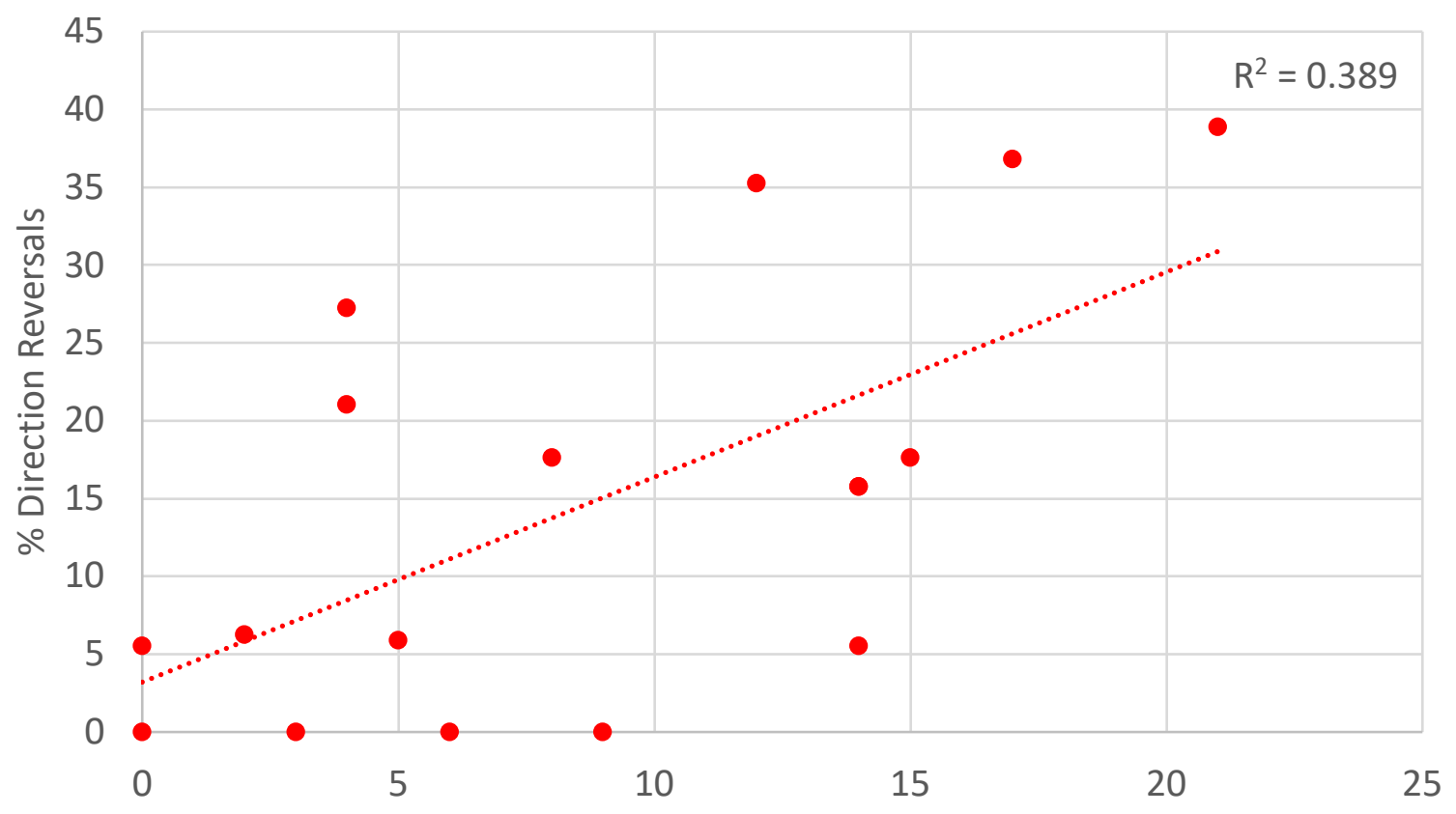

Number of SCAT Symptoms

Figure 9. Initial relationship between the \% of trials in the CMI condition resulting in a direction reversal and the number of SCAT symptoms. Following the program, there was no longer a relationship between the two. 


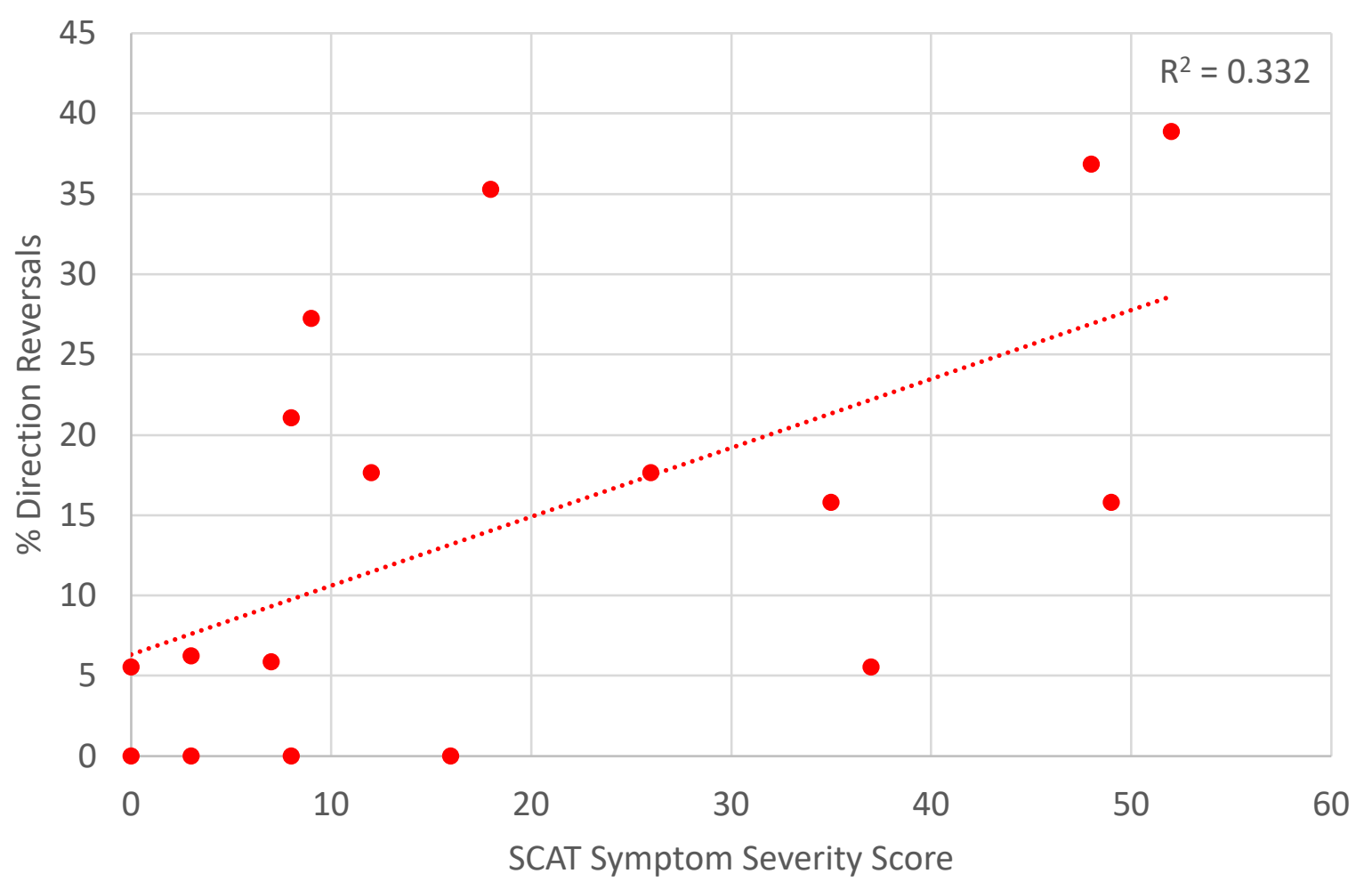

Figure 10. Initial relationship between the $\%$ of trials in the CMI condition resulting in a direction reversal and the SCAT symptom severity score. Following the program, there was no longer a relationship between the two. 


\section{Chapter 3: Discussion \& Conclusions}

\section{Discussion}

The current study investigated the resolution of rule-based, complex visuomotor task performance in individuals affected by concussion and healthy controls in working-aged adults. Our findings indicate that (1) concussion impairs visuomotor skill performance, (2) the performance of complex, rule-based tasks can be improved over the course of a recovery program, and (3) stronger recovery in females suggests sex-related differences in the brain networks controlling skilled performance, and the effect of injury on these networks. Acutely concussed participants demonstrated visuomotor performance deficits compared to healthy controls and individuals with a history of concussion prior to the concussion-management program, though these deficits recovered specifically in this group over the course of the program. We suggest that these deficits are related to disruptions in frontoparietal-cerebellar networks, with recovery of these brain networks occurring throughout the course of the program. Understanding and identifying the neural correlates of visuomotor performance deficits and recovery requires further study. Future work should utilize imaging and neurophysiological techniques, such as fMRI, EEG, and fNIRS, to provide insight into possible alterations of brain structure, function, and activity in concussed individuals. It is likewise crucial that these studies include participants with a various number of concussions. This would allow a better understanding of the neurological effects of multiple concussions on CMI and its associated networks, and would provide further insight into how brain activity and functional networks may be altered with repetitive head injury. Furthermore, given the results of the study and the increasing awareness of the effect of sex on symptomatology and recovery of mild brain injury, the use of endocrine measures, including the performance of hormone assays, may provide further insight into existing sex differences in the neurorehabilitation of mild brain injury, and may further establish a relationship between hormone levels and brain function.

There exists limited concussion rehabilitation research, particularly in working-aged adults. Based on the findings from this study, future studies should include a delayed-treatment control group, in order to identify whether observed improvements in visuomotor performance are related to OMT, and as such to determine whether this may be one form of evidence-based treatment available for those who are affected by concussion. Including a control group will thus add evidence for evidence-based practices when it comes to the scarce rehabilitation treatments 
for individuals with concussion. If the incorporation of OMT into an active recovery program is revealed to be effective in the neurorehabilitation of brain injury, it can be life-changing: functional independence in the face of brain injury may be preserved or maintained.

Finally, recovery following concussion is typically studied in youth and young adult athletes. Our study is one of the few investigating concussion recovery in working-aged adults in a non-athlete population. The present findings provide evidence for general improvement of visuomotor skill performance, and further demonstrate that CMI recovery is possible in a wide age range following concussion. In fact, we demonstrate that in working-aged adults, age is positively related to improved performance of complex, rule-based tasks. Further studies are needed to better understand this relationship and the detailed neural mechanisms involved in cognitive-motor integration following concussion in this population. Future work will increase the present age range, thus helping close the knowledge gap in a broader demographic sample.

\section{Strengths and limitations}

The current study investigated changes in visuomotor performance in individuals who are acutely concussed, those with a history of concussion, and healthy controls. Individuals who are acutely concussed or have a history of concussion are rarely studied simultaneously. As concussion is a heterogeneous condition, a key strength of this study was to investigate performance differences between these 2 groups separately and healthy controls. In addition, studies investigating concussion typically examine elite athletes. The inclusion of participants from the community who engage in all exercise levels in the current study indicates that the findings may be more generalizable than studies only considering elite athletes.

When interpreting the results of this study, it is important to acknowledge that the assessment of past concussion history was based on self-report. Therefore, potential errors are possible due to imprecise memory. Moreover, not all individuals seek medical care in the face of concussion, and consequently may not receive a concussion diagnosis. Thus, individuals who were placed in the healthy group may have belonged in the concussion or concussion history groups. In addition, a larger sample would be helpful for investigating the relationship between sex-related differences and concussion. Finally, the lack of a control group or a group receiving an alternate treatment excludes the ability to examine whether observed differences in this study were associated with OMT. 


\section{Conclusions}

The results of the present study demonstrate an overall improvement of visuomotor performance over the course of an active recovery program involving OMT, indicating that skilled performance can be improved. Moreover, prior to the program, individuals with an acute concussion showed distinct impairments in the performance of CMI tasks, though greater improvements in visuomotor tasks occurred in individuals with a concussion history compared to healthy controls. Taken together, this suggests that eye-hand coordination is impaired following mild brain injury, though recovery is possible and may be related to age. Additionally, the results suggest that underlying brain networks controlling simultaneous thought and action may differ between sexes. Overall, these findings contribute to present knowledge about motor performance on tasks requiring rule integration. 


\section{References}

Baker, C. S., \& Cinelli, M. E. (2014). Visuomotor deficits during locomotion in previously concussed athletes 30 or more days following return to play. Physiological Reports, 2(12). https://doi.org/10.14814/phy2.12252

Baltazar, G. A., Kolwitz, C., Petrone, P., Stright, A., \& Joseph, D. (2020). Osteopathic Manipulative Treatment Relieves Post-concussion Symptoms in a Case of Polytrauma. Cureus. https://doi.org/10.7759/cureus. 7317

Barkhoudarian, G., Hovda, D. A., \& Giza, C. C. (2011). The Molecular Pathophysiology of Concussive Brain Injury. Clinics in Sports Medicine, 30(1). https://doi.org/10.1016/j.csm.2010.09.001

Barlow, K. M., Brooks, B. L., MacMaster, F. P., Kirton, A., Seeger, T., Esser, M., Crawford, S., Nettel-Aguirre, A., Zemek, R., Angelo, M., Kirk, V., Emery, C. A., Johnson, D., Hill, M. D., Buchhalter, J., Turley, B., Richer, L., Platt, R., Hutchison, J., \& Dewey, D. (2014). A double-blind, placebo-controlled intervention trial of 3 and $10 \mathrm{mg}$ sublingual melatonin for post-concussion syndrome in youths (PLAYGAME): study protocol for a randomized controlled trial. Trials, 15(1). https://doi.org/10.1186/1745-6215-15-271

Barnes, B. C., Cooper, L., Kirkendall, D. T., Mcdermott, T. P., Jordan, B. D., \& Garrett, W. E. (1998). Concussion History in Elite Male and Female Soccer Players. American Journal of Sports Medicine, 26(3), 433-438.

Berz, K., Divine, J., Foss, K. B., Heyl, R., Ford, K. R., \& Myer, G. D. (2013). Sex-Specific Differences in the Severity of Symptoms and Recovery Rate following Sports-Related Concussion in Young Athletes. The Physician and Sportsmedicine, 41(2), 58-63. https://doi.org/10.3810/psm.2013.05.2015

Białuńska, A., \& Salvatore, A. P. (2017). The auditory comprehension changes over time after sport-related concussion can indicate multisensory processing dysfunctions. Brain and Behavior, 7(12). https://doi.org/10.1002/brb3.874

Black, A. M., Sergio, L. E., \& Macpherson, A. K. (2016). The Epidemiology of Concussions: Number and Nature of Concussions and Time to Recovery Among Female and Male Canadian Varsity Athletes 2008 to 2011. Clinical Journal of Sport Medicine, 27(1), 52-56. www.cjsportmed.com

Brooks, B. L., Mrazik, M., Barlow, K. M., McKay, C. D., Meeuwisse, W. H., \& Emery, C. A. (2014). Absence of Differences Between Male and Female Adolescents With Prior Sport Concussion. Journal of Head Trauma Rehabilitation, 29(3). https://doi.org/10.1097/HTR.0000000000000016

Brown, J. A., Dalecki, M., Hughes, C., Macpherson, A. K., \& Sergio, L. E. (2015). Cognitivemotor integration deficits in young adult athletes following concussion. BMC Sports Science, Medicine and Rehabilitation, 7(1). https://doi.org/10.1186/s13102-015-0019-4

Brown, K. E., Neva, J. L., Feldman, S. J., Staines, W. R., \& Boyd, L. A. (2018). Sensorimotor integration in healthy aging: Baseline differences and response to sensory training. Experimental Gerontology, 112, 1-8. https://doi.org/10.1016/j.exger.2018.08.004

Chamard, E., Lassonde, M., Henry, L., Tremblay, J., Boulanger, Y., de Beaumont, L., \& Théoret, H. (2013). Neurometabolic and microstructural alterations following a sportsrelated concussion in female athletes. Brain Injury, 27(9).

https://doi.org/10.3109/02699052.2013.794968 
Chancellor, S. E., Franz, E. S., Minaeva, O. v., \& Goldstein, L. E. (2019). Pathophysiology of Concussion. Seminars in Pediatric Neurology, 30, 14-25.

https://doi.org/10.1016/j.spen.2019.03.004

Choe, M. C. (2016). The Pathophysiology of Concussion. Current Pain and Headache Reports, 20(6). https://doi.org/10.1007/s11916-016-0573-9

Cook, M. J., Gardner, A. J., Wojtowicz, M., Williams, W. H., Iverson, G. L., \& Stanwell, P. (2020). Task-related functional magnetic resonance imaging activations in patients with acute and subacute mild traumatic brain injury: A coordinate-based meta-analysis. NeuroImage: Clinical, 25. https://doi.org/10.1016/j.nicl.2019.102129

Covassin, T., Buz Swanik, C., \& Sachs, M. L. (2003). Sex Differences and the Incidence of Concussions Among Collegiate Athletes. Journal of Athletic Training, 38(3), 238-244. www.journalofathletictraining.org

Covassin, T., Moran, R., \& Elbin, R. J. (2016). Sex Differences in Reported Concussion Injury Rates and Time Loss From Participation: An Update of the National Collegiate Athletic Association Injury Surveillance Program From 2004-2005 Through 2008-2009. Journal of Athletic Training, 51(3), 189-194. https://doi.org/10.4085/1062-6050-51.3.05

Covassin, T., Moran, R., \& Wilhelm, K. (2013). Concussion Symptoms and Neurocognitive Performance of High School and College Athletes Who Incur Multiple Concussions. The American Journal of Sports Medicine, 41(12). https://doi.org/10.1177/0363546513499230

Covassin, T., Schatz, P., \& Swanik, C. B. (2007). Sex differences in neurophysiological function and post-concussion symptoms of concussed collegiate athletes. VOLUME, 61(2), 345-351. https://doi.org/10.1227/01.NEU.0000279972.95060.CB

Covassin, T., Stearne, D., \& Elbin, R. (2008). Concussion History and Postconcussion Neurocognitive Performance and Symptoms in Collegiate Athletes. Journal of Athletic Training, 43(2). https://doi.org/10.4085/1062-6050-43.2.119

Crutcher, M. D., Russo, G. S., Ye, S., \& Backus, D. A. (2004). Target-, limb-, and contextdependent neural activity in the cingulate and supplementary motor areas of the monkey. Experimental Brain Research, 158(3). https://doi.org/10.1007/s00221-004-1895-0

Dalecki, M., Albines, D., Macpherson, A., \& Sergio, L. E. (2016). Prolonged cognitive-motor impairments in children and adolescents with a history of concussion. Concussion, 1(3). https://doi.org/10.2217/cnc-2016-0001

Dalecki, M., Gorbet, D. J., Macpherson, A., \& Sergio, L. E. (2019). Sport experience is correlated with complex motor skill recovery in youth following concussion. European Journal of Sport Science, 19(9), 1257-1266. https://doi.org/10.1080/17461391.2019.1584249

Dalecki, M., Gorbet, D. J., \& Sergio, L. E. (2019). Development of rule-based eye-handdecoupling in children and adolescents. Child Neuropsychology, 25(8), 1-18. https://doi.org/10.1080/09297049.2019.1578342

de Beaumont, L., Brisson, B., Lassonde, M., \& Jolicoeur, P. (2007). Long-term electrophysiological changes in athletes with a history of multiple concussions. Brain Injury, 21(6). https://doi.org/10.1080/02699050701426931

de Boer, C., Echlin, H. V., Rogojin, A., Baltaretu, B. R., \& Sergio, L. E. (2018). ThinkingWhile-Moving Exercises May Improve Cognition in Elderly with Mild Cognitive Deficits: A Proof-of-Principle Study. Dementia and Geriatric Cognitive Disorders Extra, 8(2). https://doi.org/10.1159/000490173 
Echlin, H. v., Gorbet, D. J., \& Sergio, L. E. (2020). Assessment of a Cognitive-Motor Training Program in Adults at Risk for Developing Dementia. Canadian Geriatrics Journal, 23(2). https://doi.org/10.5770/cgj.23.394

Edwards, L. L., King, E. M., Buetefisch, C. M., \& Borich, M. R. (2019). Putting the "Sensory" Into Sensorimotor Control: The Role of Sensorimotor Integration in Goal-Directed Hand Movements After Stroke. Frontiers in Integrative Neuroscience, 13. https://doi.org/10.3389/fnint.2019.00016

Fino, P. C., Peterka, R. J., Hullar, T. E., Murchison, C., Horak, F. B., Chesnutt, J. C., \& King, L. A. (2017). Assessment and rehabilitation of central sensory impairments for balance in mTBI using auditory biofeedback: a randomized clinical trial. BMC Neurology, 17(1). https://doi.org/10.1186/s12883-017-0812-7

Frommer, L. J., Gurka, K. K., Cross, K. M., Ingersoll, C. D., Dawn Comstock, R., \& Saliba, S. A. (2011). Sex Differences in Concussion Symptoms of High School Athletes. Journal of Athletic Training, 46(1), 76-84. www.nata.org/jat

Gallagher, V., Kramer, N., Abbott, K., Alexander, J., Breiter, H., Herrold, A. A., Lindley, T., Mjaanes, J., \& Reilly, J. (2018). The Effects of Sex Differences and Hormonal Contraception on Outcomes after Collegiate Sports-Related Concussion. Journal of Neurotrauma, 35(11), 1242-1247. https://doi.org/10.1089/neu.2017.5453

Giza, C. C., \& Hovda, D. A. (2014). The New Neurometabolic Cascade of Concussion. Neurosurgery, 75. https://doi.org/10.1227/NEU.0000000000000505

Gorbet, Diana J., \& Sergio, L. E. (2007). Preliminary sex differences in human cortical BOLD fMRI activity during the preparation of increasingly complex visually guided movements. European Journal of Neuroscience, 25(4). https://doi.org/10.1111/j.14609568.2007.05358.x

Gorbet, Diana J., \& Sergio, L. E. (2009). The behavioural consequences of dissociating the spatial directions of eye and arm movements. Brain Research, 1284, 77-88. https://doi.org/10.1016/j.brainres.2009.05.057

Gorbet, Diana J., \& Sergio, L. E. (2018). Move faster, think later: Women who play action video games have quicker visually-guided responses with later onset visuomotor-related brain activity. PLoS ONE, 13(1). https://doi.org/10.1371/journal.pone.0189110

Gorbet, Diana J., \& Sergio, L. E. (2019). Looking up while reaching out: the neural correlates of making eye and arm movements in different spatial planes. Experimental Brain Research, 237(1), 57-70. https://doi.org/10.1007/s00221-018-5395-z

Gorbet, Diana J., \& Staines, W. R. (2011). Inhibition of contralateral premotor cortex delays visually guided reaching movements in men but not in women. Experimental Brain Research, 212(2). https://doi.org/10.1007/s00221-011-2731-y

Gorbet, Diana Judith, Mader, L. B., \& Richard Staines, W. (2010). Sex-related differences in the hemispheric laterality of slow cortical potentials during the preparation of visually guided movements. Experimental Brain Research, 202(3). https://doi.org/10.1007/s00221-0102170-1

Gorbet, D.J., \& Sergio, L. E. (2016). Don't watch where you're going: The neural correlates of decoupling eye and arm movements. Behavioural Brain Research, 298, 229-240. https://doi.org/10.1016/j.bbr.2015.11.012

Gordon, K. E., Dooley, J. M., \& Wood, E. P. (2006). Descriptive Epidemiology of Concussion. Pediatric Neurology, 34(5), 376-378. https://doi.org/10.1016/j.pediatrneurol.2005.09.007 
Granek, J. A., Pisella, L., Blangero, A., Rossetti, Y., \& Sergio, L. E. (2012). The Role of the Caudal Superior Parietal Lobule in Updating Hand Location in Peripheral Vision: Further Evidence from Optic Ataxia. PLoS ONE, 7(10). https://doi.org/10.1371/journal.pone.0046619

Granek, J. A., \& Sergio, L. E. (2015). Evidence for distinct brain networks in the control of rulebased motor behavior. Journal of Neurophysiology, 114(2), 1298-1309. https://doi.org/10.1152/jn.00233.2014

Guernsey, D. T., Leder, A., \& Yao, S. (2016). Resolution of Concussion Symptoms After Osteopathic Manipulative Treatment: A Case Report. Journal of Osteopathic Medicine, 116(3). https://doi.org/10.7556/jaoa.2016.036

Hawkins, K. M., Goyal, A. I., \& Sergio, L. E. (2015). Diffusion Tensor Imaging Correlates of Cognitive-Motor Decline in Normal Aging and Increased Alzheimer's Disease Risk. Journal of Alzheimer's Disease, 44(3). https://doi.org/10.3233/JAD-142079

Hawkins, K. M., Sayegh, P., Yan, X., Crawford, J. D., \& Sergio, L. E. (2013). Neural Activity in Superior Parietal Cortex during Rule-based Visual-motor Transformations. Journal of Cognitive Neuroscience, 25(3), 436-454. https://doi.org/10.1162/jocn_a_00318

Howell, D. R., Beasley, M., Vopat, L., \& Meehan, W. P. (2017). The Effect of Prior Concussion History on Dual-Task Gait following a Concussion. Journal of Neurotrauma, 34(4). https://doi.org/10.1089/neu.2016.4609

Hurtubise, J., Gorbet, D., Hamandi, Y., Macpherson, A., \& Sergio, L. (2016). The effect of concussion history on cognitive-motor integration in elite hockey players. Concussion, 1(3). https://doi.org/10.2217/cnc-2016-0006

Hurtubise, J. M., Gorbet, Di. J., Hynes, L. M., MacPherson, A. K., \& Sergio, L. E. (2020). White Matter Integrity and Its Relationship to Cognitive-Motor Integration in Females with and without Post-Concussion Syndrome. Journal of Neurotrauma, 37(13), 1528-1536. https://doi.org/10.1089/neu.2019.6765

Hwang, E. J., Hauschild, M., Wilke, M., \& Andersen, R. A. (2014). Spatial and Temporal EyeHand Coordination Relies on the Parietal Reach Region. Journal of Neuroscience, 34(38). https://doi.org/10.1523/JNEUROSCI.3719-13.2014

Iverson, G. L., Gaetz, M., Lovell, M. R., \& Collins, M. W. (2004). Cumulative effects of concussion in amateur athletes. Brain Injury, 18(5). https://doi.org/10.1080/02699050310001617352

Ledoux, A.-A., Tang, K., Yeates, K. O., Pusic, M. v., Boutis, K., Craig, W. R., Gravel, J., Freedman, S. B., Gagnon, I., Gioia, G. A., Osmond, M. H., \& Zemek, R. L. (2019). Natural Progression of Symptom Change and Recovery From Concussion in a Pediatric Population. JAMA Pediatrics, 173(1). https://doi.org/10.1001/jamapediatrics.2018.3820

Matur, Z., \& Öge, A. E. (2017). Sensorimotor Integration During Motor Learning: Transcranial Magnetic Stimulation Studies. Nöro Psikiyatri Arşivi, 54(4). https://doi.org/10.5152/npa.2016.18056

Merritt, V. C., Padgett, C. R., \& Jak, A. J. (2019). The Clinical Neuropsychologist A systematic review of sex differences in concussion outcome: What do we know? https://doi.org/10.1080/13854046.2018.1508616

Mullally, W. J. (2017). Concussion. The American Journal of Medicine, 130(8), 885-892. https://doi.org/10.1016/j.amjmed.2017.04.016 
Murray, E. A., Bussey, T. J., \& Wise, S. P. (2000). Role of prefrontal cortex in a network for arbitrary visuomotor mapping. In Executive Control and the Frontal Lobe: Current Issues. Springer Berlin Heidelberg. https://doi.org/10.1007/978-3-642-59794-7_13

Ono, K. E., Burns, T. G., Bearden, D. J., McManus, S. M., King, H., \& Reisner, A. (2015). SexBased Differences as a Predictor of Recovery Trajectories in Young Athletes After a Sports-Related Concussion. The American Journal of Sports Medicine, 44(3), 748-752. https://doi.org/10.1177/0363546515617746

Origenes, A. K., Alleva, J. T., \& Hudgins, T. H. (2019). Concussion rehabilitation/post concussion syndrome. Disease-a-Month, 65(10). https://doi.org/10.1016/j.disamonth.2019.02.007

Piaget, J. (1965). The stages of the intellectual development of the child. In Educational psychology in context: Readings for future teachers (4th ed., Vol. 63, pp. 98-106).

Plourde, V., Rohr, C. S., Virani, S., Bray, S., Yeates, K. O., \& Brooks, B. L. (2020). Default mode network functional connectivity after multiple concussions in children and adolescents. Archives of Clinical Neuropsychology, 35(3). https://doi.org/10.1093/arclin/acz073

Rogojin, A., Gorbet, D. J., Hawkins, K. M., \& Sergio, L. E. (2019). Cognitive-Motor Integration Performance Is Affected by Sex, APOE Status, and Family History of Dementia. Journal of Alzheimer's Disease, 71(2). https://doi.org/10.3233/JAD-190403

Sayegh, P. F., Gorbet, D. J., Hawkins, K. M., Hoffman, K. L., \& Sergio, L. E. (2017). The Contribution of Different Cortical Regions to the Control of Spatially Decoupled Eye-Hand Coordination. Journal of Cognitive Neuroscience, 29(7). https://doi.org/10.1162/jocn_a_01111

Sayegh, P. F., Hawkins, K. M., Hoffman, K. L., \& Sergio, L. E. (2013). Differences in spectral profiles between rostral and caudal premotor cortex when hand-eye actions are decoupled. Journal of Neurophysiology, 110(4). https://doi.org/10.1152/jn.00764.2012

Sayegh, P. F., Hawkins, K. M., Neagu, B., Crawford, J. D., Hoffman, K. L., \& Sergio, L. E. (2014). Decoupling the actions of the eyes from the hand alters beta and gamma synchrony within SPL. Journal of Neurophysiology, 111(11). https://doi.org/10.1152/jn.00793.2013

Sergio, L. E., Gorbet, D. J., Tippett, W. J., Yan, X., \& Neagu, B. (2009). Where what you see isn't where you get: Cortical mechanisms of vision for complex action. In M. Jenkin \& L. Harris (Eds.), Cortical mechanisms of vision (pp. 81-118). Cambridge University Press.

Sergio, Lauren E., Gorbet, D. J., Adams, M. S., \& Dobney, D. M. (2020). The Effects of Mild Traumatic Brain Injury on Cognitive-Motor Integration for Skilled Performance. Frontiers in Neurology, 11. https://doi.org/10.3389/fneur.2020.541630

Sharp, D. J., \& Jenkins, P. O. (2015). Concussion is confusing us all. Practical Neurology, 15(3), 172-186. https://doi.org/10.1136/practneurol-2015-001087

Slobounov, S., Slobounov, E., \& Newell, K. (2006). Application of virtual reality graphics in assessment of concussion. Cyberpsychology \& behavior : the impact of the Internet, multimedia and virtual reality on behavior and society, 9(2), 188-191. https://doi.org/10.1089/cpb.2006.9.188

Slobounov, S. M., Zhang, K., Pennell, D., Ray, W., Johnson, B., \& Sebastianelli, W. (2010). Functional abnormalities in normally appearing athletes following mild traumatic brain injury: a functional MRI study. Experimental Brain Research, 202(2).

https://doi.org/10.1007/s00221-009-2141-6 
Teel, E. F., Marshall, S. W., Shankar, V., McCrea, M., \& Guskiewicz, K. M. (2017). Predicting Recovery Patterns After Sport-Related Concussion. Journal of Athletic Training, 52(3). https://doi.org/10.4085/1062-6050-52.1.12

Thériault, M., de Beaumont, L., Gosselin, N., Filipinni, M., \& Lassonde, M. (2009). Electrophysiological abnormalities in well functioning multiple concussed athletes. Brain Injury, 23(11). https://doi.org/10.1080/02699050903283189

Tremblay, Sara, de Beaumont, L., Lassonde, M., \& Théoret, H. (2011). Evidence for the Specificity of Intracortical Inhibitory Dysfunction in Asymptomatic Concussed Athletes. Journal of Neurotrauma, 28(4). https://doi.org/10.1089/neu.2010.1615

Tremblay, Sebastien, Henry, L. C., Bedetti, C., Larson-Dupuis, C., Gagnon, J.-F., Evans, A. C., Théoret, H., Lassonde, M., \& Beaumont, L. de. (2014). Diffuse white matter tract abnormalities in clinically normal ageing retired athletes with a history of sports-related concussions. Brain, 137(11). https://doi.org/10.1093/brain/awu236

Virji-Babul, N., Borich, M. R., Makan, N., Moore, T., Frew, K., Emery, C. A., \& Boyd, L. A. (2013). Diffusion Tensor Imaging of Sports-Related Concussion in Adolescents. Pediatric Neurology, 48(1). https://doi.org/10.1016/j.pediatrneurol.2012.09.005

Wise, S. P., di Pellegrino, G., \& Boussaoud, D. (1996). The premotor cortex and nonstandard sensorimotor mapping. Canadian Journal of Physiology and Pharmacology, 74(4). https://doi.org/10.1139/cjpp-74-4-469

Wojcik, S. M. (2014). Predicting mild traumatic brain injury patients at risk of persistent symptoms in the Emergency Department. Brain Injury, 28(4). https://doi.org/10.3109/02699052.2014.884241

Yao, S. C., Zwibel, H., Angelo, N., Leder, A., \& Mancini, J. (2020). Effectiveness of Osteopathic Manipulative Medicine vs Concussion Education in Treating Student Athletes With Acute Concussion Symptoms. Journal of Osteopathic Medicine, 120(9). https://doi.org/10.7556/jaoa.2020.099 


\section{Contributions}

Nicole Smeha was responsible for data scoring, data analysis design and execution, and primary writing of the manuscript. Ravneet Kalkat performed the data collection and provided feedback on the written manuscript. Loriann Hynes was involved in the experiment design and assisted with the written manuscript. Lauren Sergio was involved in the experiment design, assisted with data analysis, and edited the manuscript. Leah Henderson was involved in the intervention component of the project and assisted with participant recruitment. 


\section{Appendix}

A : Health Questionnaire

\section{Health Questionnaire}

Step 1: Athlete Background - SCAT5 (included)

Pretest Intake Questionnaire - BrDI, HRV and Doppler study

(The information received will remain confidential)

ID:

Age: Height:

Weight:

Dominant Hand: LEFT or RIGHT or BOTH

Level of Education:

Ethnicity:

Sport(s) Played (recreational or competitive):

How long did you play your sport(s):

1. Do you currently have a concussion? (an impact to the head which MAY have resulted in: headaches, loss of consciousness, confusion, amnesia, dizziness, nausea, etc)

YES or NO (if answer is NO please proceed to question 2)

a) Date of concussion:

b) Did you lose consciousness? For how long?

c) Please list any current signs and symptoms:

2. Have you previously had any concussions (an impact to the head which MAY have resulted in: headaches, loss of consciousness, confusion, amnesia, dizziness, nausea, etc)?

YES or NO (if answer is NO please proceed to question 3)

a) How many?

b) Did you lose consciousness? For how long?

c) Date(s) and time out before returning to play:

d) Did you receive treatment for your concussion? YES or NO Type of treatment received:
Date of Test:

Neck Circumference:

DOB:

Sex: Male or Female or Other

Work Full Time / Part Time / Neither:

Occupation: 
3. Have you ever been hospitalized for a head injury? YES or NO

4. Current medications? If yes, please list:

5. Do you smoke? YES or NO

a) Do you smoke: Cigarettes, Cannabis, Both or Other:

b) How often do you smoke the above mentioned substance(s) per day, week or month?

6. In the last 2 hours have you consumed/inhaled
a. any stimulants such as caffeine (coffee) or nicotine (smoking)? YES or NO
b. any depressants such as marijuana or alcohol?
YES or NO

7. Have you been diagnosed with any neurological disorders? YES or NO

What disorder?

8. Have you been diagnosed with any of the following:

\begin{tabular}{|l|l|l|l|}
\hline \multicolumn{1}{|c|}{ Disorder } & YES & NO & \\
\hline Diabetes & & & \\
\hline Cancer & & & \\
\hline Autoimmune Disorder \\
(Rheumatoid Arthritis, Lupus)
\end{tabular}


9. Has anyone in your family been diagnosed with any of the following:

\begin{tabular}{|c|c|c|c|}
\hline Disorder & YES & $\mathrm{NO}$ & Diagnosis \\
\hline Diabetes & & & \\
\hline Cancer & & & \\
\hline $\begin{array}{l}\text { Autoimmune Disorder } \\
\text { (Rheumatoid Arthritis, } \\
\text { Lupus) }\end{array}$ & & & \\
\hline $\begin{array}{l}\text { Cerebrovascular Disease } \\
\text { (Stroke, Aneurism) }\end{array}$ & & & \\
\hline $\begin{array}{l}\text { Cardiovascular Disease } \\
\text { (Heart attack, Heart failure) }\end{array}$ & & & \\
\hline Hypertension & & & \\
\hline Dementia & & & \\
\hline
\end{tabular}

10. Do you have a computer (YES or NO) or a tablet (YES or NO) at home?

$\begin{array}{ll}\text { How often do you use your computer? } & \text { (all the time / often / sometimes / rarely / never) } \\ \text { How often do you use your tablet? } & \text { (all the time / often / sometimes / rarely / never) }\end{array}$

11. Do you do puzzles? YES or NO

(all the time / often / sometimes / rarely / never)

12. Do you play video games? YES or NO (all the time / often / sometimes / rarely / never)

a. What type of games do you typically play? ACTION (time pressure) or NON-ACTION

b. How would you rate your skill compared to your peers? (Low / Intermediate / High) 
THE FOLLOWING IS A LIST OF ACTIVITIES THAT PEOPLE MAY PARTICIPATE IN. PLEASE INDICATE THE FREQUENCY (IN DAYS PER WEEK) THAT YOU TYPICALLY PARTICIPATE IN THESE ACTIVITES.

FOR EACH ITEM CHOOSE FROM ONE OF THE FOLLOWING ALTERNATIVES:

\begin{tabular}{|c|c|c|c|c|c|}
\hline NEV & & $\begin{array}{l}\text { RARELY } \\
\text { (1 DAY/ } \\
\text { WEEK) }\end{array}$ & $\begin{array}{l}\text { SOMETIMES } \\
\text { (2 DAYS/ } \\
\text { WEEK) }\end{array}$ & $\begin{array}{l}\text { FAIRLY OFTEN } \\
(3-4 \text { DAYS/ } \\
\text { WEEK) }\end{array}$ & $\begin{array}{l}\text { VERY OFTEN } \\
\text { (5-7 DAYS/ } \\
\text { WEEK) }\end{array}$ \\
\hline & $\underline{0}$ & $\underline{1}$ & $\underline{2}$ & $\underline{3}$ & $\underline{4}$ \\
\hline $\begin{array}{l}\text { WATCHING TV OR } \\
\text { MOVIES }\end{array}$ & 0 & 1 & 2 & 3 & 4 \\
\hline READING & 0 & 1 & 2 & 3 & 4 \\
\hline $\begin{array}{l}\text { SOCIALIZING (E.G. } \\
\text { PLAYING CARDS, TALKING } \\
\text { TO FRIENDS, ETC.) }\end{array}$ & 0 & 1 & 2 & 3 & 4 \\
\hline PLAYING REC SPORTS 0 & & 1 & 2 & 3 & 4 \\
\hline $\begin{array}{l}\text { PLAYING } \\
\text { COMPETITIVE SPORTS }\end{array}$ & 0 & 1 & 2 & 3 & 4 \\
\hline $\begin{array}{l}\text { PLAYING VIDEO/ } \\
\text { COMPUTER GAMES }\end{array}$ & 0 & 1 & 2 & 3 & 4 \\
\hline $\begin{array}{l}\text { WALKING (AT LEAST } \\
25 \text { MINUTES) }\end{array}$ & 0 & 1 & 2 & 3 & 4 \\
\hline LISTENING TO MUSIC 0 & & 1 & 2 & 3 & 4 \\
\hline $\begin{array}{l}\text { EXERCISING AT A } \\
\text { GYM }\end{array}$ & 0 & 1 & 2 & 3 & 4 \\
\hline DOING NON-LABOUR & 0 & 1 & 2 & 3 & 4 \\
\hline
\end{tabular}




$\begin{array}{llllll}\begin{array}{l}\text { DOING LABOUR } \\ \text { WORK (E.G. LANDSCAPING }\end{array} & 0 & 1 & 2 & 3 & 4 \\ \begin{array}{l}\text { SHOVELING, PAINTING, ETC. } \\ \text { PAID OR VOLUNTEER) }\end{array} & & & & \\ \text { RUNNING/JOGGING } & 0 & 1 & 2 & 3 & 4 \\ \begin{array}{l}\text { 13. PUZZLES, ARTS \& } \\ \text { CRAFTS (E.G. KNITTING, }\end{array} & 0 & 1 & 2 & 3 & 4 \\ \text { CROSSWORDS, ETC.) } & & & & \end{array}$

\section{LAST QUESTION! FEMALES ONLY}

1. Menstrual Cycle:

a. Flow: Light or Regular or Heavy

b. Length of Cycle:

c. Contraceptive Use: Birth Control or IUD or Other

d. Peri-menopausal or Post- menopausal* or Not Applicable

*post- menopausal is defined as having no period for the past 12 months

e. Circle all that apply: PCOS, Endometriosis, Fibroids, Cysts 


\section{B: SCAT5 - SYMPTOM EVALUATION}

\section{STEP 2: Symptom Evaluation - SCAT5}

You should score yourself on the following symptoms, based on how you feel now.

\begin{tabular}{|c|c|c|c|c|c|c|c|}
\hline & none & & iild & & erate & & \\
\hline Headache & 0 & 1 & 2 & 3 & 4 & 5 & 6 \\
\hline Pressure in head & 0 & 1 & 2 & 3 & 4 & 5 & 6 \\
\hline Neck Pain & 0 & 1 & 2 & 3 & 4 & 5 & 6 \\
\hline Nausea or vomiting & 0 & 1 & 2 & 3 & 4 & 5 & 6 \\
\hline Dizziness & 0 & 1 & 2 & 3 & 4 & 5 & 6 \\
\hline Blurred Vision & 0 & 1 & 2 & 3 & 4 & 5 & 6 \\
\hline Balance Problems & 0 & 1 & 2 & 3 & 4 & 5 & 6 \\
\hline Sensitivity to light & 0 & 1 & 2 & 3 & 4 & 5 & 6 \\
\hline Sensitivity to noise & 0 & 1 & 2 & 3 & 4 & 5 & 6 \\
\hline Feeling slowed down & 0 & 1 & 2 & 3 & 4 & 5 & 6 \\
\hline Feeling like "in a fog" & 0 & 1 & 2 & 3 & 4 & 5 & 6 \\
\hline "Don't feel right" & 0 & 1 & 2 & 3 & 4 & 5 & 6 \\
\hline Difficulty concentrating & 0 & 1 & 2 & 3 & 4 & 5 & 6 \\
\hline Difficulty remembering & 0 & 1 & 2 & 3 & 4 & 5 & 6 \\
\hline Fatigue or low energy & 0 & 1 & 2 & 3 & 4 & 5 & 6 \\
\hline Confusion & 0 & 1 & 2 & 3 & 4 & 5 & 6 \\
\hline Drowsiness & 0 & 1 & 2 & 3 & 4 & 5 & 6 \\
\hline More emotional & 0 & 1 & 2 & 3 & 4 & 5 & 6 \\
\hline Irritability & 0 & 1 & 2 & 3 & 4 & 5 & 6 \\
\hline Sadness & 0 & 1 & 2 & 3 & 4 & 5 & 6 \\
\hline Nervous or Anxious & 0 & 1 & 2 & 3 & 4 & 5 & 6 \\
\hline Trouble falling asleep (if applicable) & 0 & 1 & 2 & 3 & 4 & 5 & 6 \\
\hline Total number of symptoms & \multicolumn{5}{|c|}{ Out of 22} & & \\
\hline
\end{tabular}




\begin{tabular}{|l|l|l|l|}
\hline Symptom severity & \multicolumn{1}{|c|}{ Out of 132 } & \multicolumn{2}{|c|}{} \\
\hline $\begin{array}{l}\text { Do your symptoms get worse with } \\
\text { physical activity? }\end{array}$ & Y & N \\
\hline $\begin{array}{l}\text { Do your symptoms get worse with } \\
\text { mental activity? }\end{array}$ & & Y & N \\
\hline $\begin{array}{l}\text { If } 100 \% \text { is feeling perfectly normal, } \\
\text { what percent do you feel? }\end{array}$ & & & \\
\hline
\end{tabular}

If not $100 \%$, why?

Echemendia, R. J., Meeuwisse, W., McCrory, P., Davis, G. A., Putukian, M., Leddy, J., . . Raftery, M. (2017). The sport concussion assessment tool 5th edition (SCAT5). Br J Sports Med, bjsports-2017-097506. 